\title{
Two-Point DTC Sets for Neufang's Convolution Algebra of Nuclear Operators
}

\section{Matthew Mazowita}

\author{
A thesis submitted to \\ The Faculty of Graduate Studies and Research \\ at Carleton University \\ in partial fulfillment of the requirements of the degree of \\ Master of Science \\ from the
}

Ottawa-Carleton Institute of Mathematics and Statistics

(C)2007 Matthew Mazowita 


$\begin{array}{ll}\begin{array}{l}\text { Library and } \\ \text { Archives Canada }\end{array} & \begin{array}{l}\text { Bibliothèque et } \\ \text { Archives Canada }\end{array} \\ \begin{array}{l}\text { Published Heritage } \\ \text { Branch }\end{array} & \begin{array}{l}\text { Direction du } \\ \text { Patrimoine de l'édition }\end{array} \\ \begin{array}{l}\text { 395 Wellington Street } \\ \text { Ottawa ON K1A 0N4 } \\ \text { Canada }\end{array} & \begin{array}{l}\text { 395, rue Wellington } \\ \text { Ottawa ON K1A ON4 }\end{array} \\ \text { Canada }\end{array}$

Your file Votre référence

ISBN: 978-0-494-36848-0

Ourfile Notre référence

ISBN: 978-0-494-36848-0

\section{NOTICE:}

The author has granted a nonexclusive license allowing Library and Archives Canada to reproduce, publish, archive, preserve, conserve, communicate to the public by telecommunication or on the Internet, loan, distribute and sell theses worldwide, for commercial or noncommercial purposes, in microform, paper, electronic and/or any other formats.

The author retains copyright ownership and moral rights in this thesis. Neither the thesis nor substantial extracts from it may be printed or otherwise reproduced without the author's permission.
AVIS:

L'auteur a accordé une licence non exclusive permettant à la Bibliothèque et Archives Canada de reproduire, publier, archiver, sauvegarder, conserver, transmettre au public par télécommunication ou par l'Internet, prêter, distribuer et vendre des thèses partout dans le monde, à des fins commerciales ou autres, sur support microforme, papier, électronique et/ou autres formats.

L'auteur conserve la propriété du droit d'auteur et des droits moraux qui protège cette thèse. $\mathrm{Ni}$ la thèse ni des extraits substantiels de celle-ci ne doivent être imprimés ou autrement reproduits sans son autorisation.
In compliance with the Canadian

Privacy Act some supporting forms may have been removed from this thesis.

While these forms may be included in the document page count, their removal does not represent any loss of content from the thesis.
Conformément à la loi canadienne sur la protection de la vie privée, quelques formulaires secondaires ont été enlevés de cette thèse.

Bien que ces formulaires aient inclus dans la pagination, il n'y aura aucun contenu manquant.

\section{Canadä}




\begin{abstract}
Neufang showed in [28] that the algebra $\mathcal{N}\left(L_{p}(G)\right)$ of nuclear operators with a convolution product is left but not right strongly Arens irregular for a large class of locally compact groups $G$. We study this algebra on discrete groups. We give representations $\Gamma$ of $\mathcal{N}\left(\ell_{p}(G)\right)^{* *}$ and $\tilde{\gamma}$ of $\operatorname{LUC}(G)^{*}$ on $B\left(\ell_{p}(G)\right)$ and show that the left topological centre condition for $m \in \mathcal{N}\left(\ell_{p}(G)\right)^{* *}$ is equivalent to $\Gamma(m) \tilde{\gamma}(f)=\tilde{\gamma}(f) \Gamma(m)$ $\forall f \in \mathrm{LUC}(G)^{*}$. Using this result and an automatic normality result inspired by [21], we show that $\mathcal{N}\left(\ell_{p}(G)\right)$ is left strongly Arens irregular (extending Neufang's result) and give 2-point left DTC (determining for the topological centre) sets for $\mathcal{N}\left(\ell_{p}(G)\right.$ ) if $G$ is abelian. We introduce a new notion of DTC in terms of representations for which we have a 2-point left set for $\mathcal{N}\left(\ell_{p}(G)\right)$ for nonabelian $G$. We are able to lift right DTC sets (in the usual sense) for $\ell_{1}(G)$ (e.g. the 2-point set from [11]) to $\mathcal{N}\left(\ell_{p}(G)\right)$
\end{abstract}




\section{Acknowledgements}

First and foremost, I would like to thank my supervisor, Prof. Matthias Neufang, for his support, advice, ideas, and conversations, and for being essentially everything I hope to be in my life.

I am grateful to the analysis research group at the Ottawa-Carleton Institute of Mathematics and Statistics, especially Prof. Vladimir Pestov for his continuing advice and Prof. Thierry Giordano for first putting me on the road to functional analysis.

I have to thank my parents for the obvious reasons and my girlfriend, Andrea McEwan, for not breaking up with me even after the nights I would wake her up at 3 a.m. to tell her that I had a great idea and then again at 4 a.m. to tell her that it was wrong.

I would also like to thank my closest mathematical friends, Jesse Collingwood, Chris Dionne, Samantha Marion, Slava Pestov, and Andrew Staal, for making mathematics a social activity.

Finally, I would like to thank Leonard Cohen, Brian Eno, Leslie Feist, and Tom Waits for keeping me sane while I wrote this thesis, a nontrivial task. 
Perhaps I could best describe my experience of doing mathematics in terms of entering a dark mansion. One goes into the first room and it's dark, completely dark, one stumbles around bumping into the furniture and then gradually you learn where each piece of furniture is, and finally after six months or so you find the light switch, you turn it on, suddenly it's all illuminated, you can see exactly where you were.

Andrew Wiles

I can only describe my experience of doing functional analysis in terms of entering a dark, infinite-dimensional warehouse. One runs as fast as one can in one direction, flailing your arms wildly in the hope that you'll bump into something, anything. The existence of a light switch is independent of ZFC.

Matthew Mazowita

Young man, in mathematics, you don't understand things. You just get used to them. 


\section{Contents}

Abstract $\quad$ ii

Introduction $\quad 1$

1 Preliminaries 5

1.1 Banach spaces, algebras, and modules . . . . . . . . . . 6

1.2 Linear operators . . . . . . . . . . . . . . . . . . . . 8

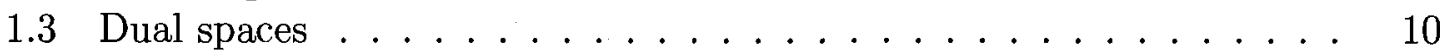

1.4 Banach space topologies . . . . . . . . . . . . . . . . 11

1.5 Abstract harmonic analysis . . . . . . . . . . . . . 13

2 Second Duals of Banach Algebras 17

2.1 Second duals . . . . . . . . . . . . . . . . . 18

2.2 Arens products . . . . . . . . . . . . . . . . 21

2.3 Topological centres and DTC sets . . . . . . . . . . 23

3 Tensor Products of Banach Spaces $\quad 27$

3.1 The algebraic tensor product . . . . . . . . . . 28

3.2 The injective tensor product . . . . . . . . . . . . . 29

3.3 The projective tensor product . . . . . . . . . . . . . 31

3.4 The Approximation and Radon-Nikodym Properties . . . . . . . . . 33

4 Nuclear Operators $\quad 36$

4.1 The convolution algebra of nuclear operators . . . . . . . . . 37

$4.2 B\left(L_{p}(G)\right)$ as a Banach module . . . . . . . . . . . . . . . 39

$4.3 G$-continuous operators . . . . . . . . . . . . 42

5 Spiral Nebulae $\quad 49$

5.1 Spiral nebulae on von Neumann algebras . . . . . . . . . . . . 50

5.2 Spiral nebulae on $B\left(L_{p}(G)\right) \ldots \ldots \ldots \ldots$. . . . . . . . . . . . . . . . . . 51

5.3 The automatic normality result .............. 53 
6 DTC Sets for Neufang's Algebra $\quad 64$ 6.1 The topological centres of $\mathcal{N}\left(L_{p}(G)\right)^{* *} \ldots \ldots \ldots \ldots \ldots 6$

6.2 A representation of $\operatorname{LUC}(G)^{*} \ldots \ldots \ldots \ldots \ldots$. . . . . . 67

6.3 The left centre condition and commuting representations . . . . . 73

6.4 Left strong Arens irregularity of $\mathcal{N}\left(\ell_{p}(G)\right)$ and left DTC sets . . . . 79

6.5 A right $\mathrm{DTC}$ set $\ldots \ldots \ldots \ldots \ldots \ldots \ldots$

$\begin{array}{ll}\text { References } & 87\end{array}$ 


\section{Introduction}

Every Banach space $X$ embeds $w^{*}$-densely in its second dual $X^{* *}$, by which we identify $X \subseteq X^{* *}$. So it is natural to ask if the product on a Banach algebra can be extended to its second dual. Richard Arens showed in 1951 in [1] and [2] that this can be done in two canonical ways yielding what are now called the first and second (or left and right) Arens products, which we denote by $\square$ and $\diamond$. These products are defined via module actions but have a nice topological characterization in terms of iterated $w^{*}$-limits.

The question "When do the two products coincide?" leads to the definition of the topological centres of $\mathfrak{A}$ :

$$
\begin{aligned}
& \mathcal{Z}_{t}^{1}\left(\mathfrak{A}^{* *}\right):=\left\{m \in \mathfrak{A}^{* *} \mid m \square n=m \diamond n \quad \forall n \in \mathfrak{A}^{* *}\right\} \\
& \mathcal{Z}_{t}^{2}\left(\mathfrak{A}^{* *}\right):=\left\{n \in \mathfrak{A}^{* *} \mid m \square n=m \diamond n \quad \forall m \in \mathfrak{A}^{* *}\right\} .
\end{aligned}
$$

The centres are norm-closed subalgebras of $\mathfrak{A}^{* *}$ (with $\square$ or $\diamond$ ) which contain $\mathfrak{A}$ and yield a classification of Banach algebras in terms of Arens regularity. A Banach algebra $\mathfrak{A}$ is Arens regular if $\mathcal{Z}_{t}^{1}\left(\mathfrak{A}^{* *}\right)=\mathfrak{A}^{* *}\left(\Longleftrightarrow \mathcal{Z}_{t}^{2}\left(\mathfrak{A}^{* *}\right)=\mathfrak{A}^{* *}\right)$ and left (respectively right) Arens irregular if $\mathcal{Z}_{t}^{1}\left(\mathfrak{A}^{* *}\right)$ (resp. $\left.\mathcal{Z}_{t}^{2}\left(\mathfrak{A}^{* *}\right)\right) \subsetneq \mathfrak{A}^{* *}$. If the left/right centre is minimal (i.e. equal to $\mathfrak{A}$ ), the algebra is called left/right strongly Arens irregular. 
It is by now a classical result that every $\mathrm{C}^{*}$-algebra (indeed, any subalgebra of $B(\mathcal{H}))$ is Arens regular [6]. On the other hand, the group algebra $L_{1}(G)$ is strongly Arens irregular for every locally compact group $G$ [25]. The first example of a Banach algebra which has one centre minimal but not the other is the algebra $\left(\mathcal{N}\left(L_{p}(G)\right), *\right)$ of nuclear operators on $L_{p}(G)$ with a convolution product (in the sense that the map $\pi: \mathcal{N}\left(L_{p}(G)\right) \rightarrow L_{1}(G)$, the preadjoint of the embedding $L_{\infty}(G) \hookrightarrow B\left(L_{p}(G)\right)$, is an algebra homomorphism), due to Neufang. He shows in [28] that it is left but not right strongly Arens irregular if $G$ is locally compact non-compact and second countable.

Recently there has been work in finding small subsets of the second dual which are determining for the topological centre (DTC), in the sense that $\mathfrak{D}$ is left DTC for $\mathfrak{A}$ if

$$
m \square d=m \diamond d \quad \forall d \in \mathfrak{D} \quad \Rightarrow \quad m \in \mathcal{Z}_{t}^{1}\left(\mathfrak{A}^{* *}\right)
$$

Surprisingly, sets consisting of two points are sufficient for $\operatorname{LUC}(G)^{*}([29]$ and [11]) and one point is sufficient for $\ell_{1}\left(\mathbb{F}_{2}\right)^{* *}([11]$ again)!

In this thesis, we show that if $G$ is infinite discrete then the left topological centre condition on the second dual of Neufang's algebra $\left(\mathcal{N}\left(\ell_{p}(G)\right), *\right)$ is equivalent to a representation $\Gamma$ of $\mathcal{N}\left(\ell_{p}(G)\right)^{* *}$ commuting with a representation $\tilde{\gamma}$ of $\operatorname{LUC}(G)^{*}$. Combining this with an automatic normality result coming from an obscure construction inspired by Hofmeier and Wittstock's work in [21] yields that there are two points $\psi_{ \pm} \in \operatorname{LUC}(G)^{*}$ s.t. if $\Gamma(m) \tilde{\gamma}\left(\psi_{ \pm}\right)=\tilde{\gamma}\left(\psi_{ \pm}\right) \Gamma(m)$ then $m \in \mathcal{Z}_{t}^{1}\left(\mathcal{N}\left(\ell_{p}(G)\right)^{* *}\right)$. From this we deduce that $\mathcal{N}\left(\ell_{p}(G)\right)$ is left strongly Arens irregular (extending Neufang's result) and that $\mathcal{N}\left(\ell_{p}(G)\right)$ admits 2-point left DTC set if $G$ is also abelian. We define a new notion of being determining for the topological centres in terms of commuting representations for which $\psi_{ \pm}$are left "DTC" set for $\mathcal{N}\left(\ell_{p}(G)\right)$ even if $G$ is nonabelian. 
We are able to lift right DTC sets for $\ell_{1}(G)$ to right DTC sets for $\mathcal{N}\left(\ell_{p}(G)\right)$ easily without relying on representation techniques. Combining this with the 2-point right DTC set for $\ell_{1}(G)$ (where $G$ is infinite discrete) from [11], we deduce that $\mathcal{N}\left(\ell_{p}(G)\right.$ ) also has a 2-point right DTC set.

Except for Section 2.3, Chapters 1 through 3 consist of standard material. Chapter 1 is a sketch of the background necessary for the rest of this thesis (assuming the prerequisites, see below). In Chapter 2 we collect the important results for the second duals of Banach spaces and algebras before moving on to Arens products, topological centres, and DTC sets. Chapter 3 gives the results on tensor products of Banach spaces needed to understand why $\mathcal{K}\left(L_{p}(G)\right)^{*}=\mathcal{N}\left(L_{p}(G)\right)$ and $\mathcal{N}\left(L_{p}(G)\right)^{*}=B\left(L_{p}(G)\right)$. The reader with a good knowledge of functional and abstract harmonic analysis should only have to read Section 2.3, since it contains the less-than-standard theory of Arens products and some new definitions.

Chapters 4 through 6 are the meat of this thesis, consisting of material from research articles and new results. Chapter 4 introduces Neufang's convolution algebra of nuclear operators from [28], the main object of study of this thesis, and Bekka's $G$ continuous operators. The new results in this chapter are extensions of results known only in the von Neumann or $\mathrm{C}^{*}$-algebra case. Chapter 5 is the requisite technical chapter of the thesis; there we construct the obscure object inspired by Hofmeier and Wittstock and use it to prove an automatic normality result which we lean on heavily in Chapter 6. Chapter 6 contains the main results of the thesis, in particular the left strong Arens irregularity of $\mathcal{N}\left(\ell_{p}(G)\right)$ and left and right DTC sets for $\mathcal{N}\left(\ell_{p}(G)\right)$. All of the results of Chapters 5 and 6 are new except for those in Sections 5.1 and 6.1. Section 5.1 describes Hofmeier and Wittstocks's inspirational construction and the results of Section 6.1 are due to Neufang and Auger [3]. 


\section{Prerequisites}

We assume standard knowledge of linear algebra (see Halmos [18]), general topology (see the classic Kelley [23] or the more modern Willard [35]), and measure theory (see Cohn [7]). While we present the basic definitions and results of functional and abstract harmonic analysis, some familiarity is assumed. For Banach space theory, we refer the reader to the terse and advanced Fabian et al [13] or the prolix and elementary Megginson [26]. For the theory of Banach algebras, see either of the tomes Dales [8] or Palmer [31]. For tensor products of Banach spaces, the only penetrable reference is the fortunately excellent Ryan [33]. For abstract harmonic analysis, see the biblical two volumes of Hewitt and Ross [19] and [20] or the introductory Folland [14].

\section{Notation and conventions}

The symbol ":=" means "is defined to be equal to". The symbol "=" denotes equal-

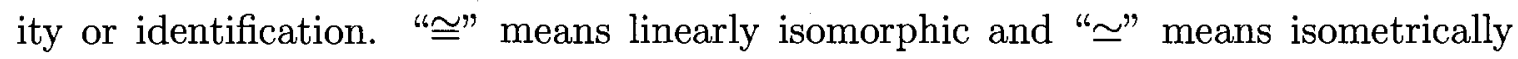
isomorphic.

If $p \in[1, \infty]$ then $q$ is the unique (extended real) number in $[1, \infty]$ s.t. $\frac{1}{p}+\frac{1}{q}=1$. By convention, $\frac{1}{\infty}=0$.

For a function $f$ defined on a locally compact group,

$$
\widetilde{f}(g):=f\left(g^{-1}\right) \quad \text { and } \quad \widetilde{f}(g):=\Delta(g)^{-1} f\left(g^{-1}\right)
$$

where $\Delta$ is the modular function of the group.

Left translations $L_{t}$ for group elements $t$ and conjugation $\operatorname{Ad} a$ by invertible $a \in \mathfrak{A}$ (a Banach algebra) are defined so that they are antirepresentations. 


\section{Chapter 1}

\section{Preliminaries}

This chapter is an incomplete collection the basic definitions and results we will need in the later chapters. The reader with a good knowledge of functional and abstract harmonic analysis can probably skip this chapter entirely. On the other hand, it certainly would not suffice to introduce a layman to these disciplines. It is included here for the benefit of those who have had some exposure to this material and as a refresher for the better acquainted. 
CHAPTER 1. PRELIMINARIES

\subsection{Banach spaces, algebras, and modules}

Definition 1.1.1. A Banach space $X$ is a complete normed complex vector space. The unit ball of $X$ is

$$
X_{1}:=\{x \in X:\|x\| \leq 1\} .
$$

Examples. Hilbert spaces, $L_{p}$ spaces, and for any topological space $T$, both

$$
\begin{gathered}
C_{b}(T):=\left\{\text { continuous } f: T \rightarrow \mathbb{C}\left|\sup _{t \in T}\right| f(t) \mid<\infty\right\} \quad \text { and } \\
C_{0}(T):=\left\{\text { continuous } f: T \rightarrow \mathbb{C} \mid \forall \varepsilon>0 \exists \text { a compact } K \subseteq T \text { s.t. }|f|_{T \backslash K} \mid<\varepsilon\right\}
\end{gathered}
$$

with the supremum norm

$$
\|f\|_{\infty}:=\sup _{t \in T}|f(t)| .
$$

Definition 1.1.2. A Banach algebra $\mathfrak{A}$ is a Banach space with an associative bilinear multiplication (in general, written as juxtaposition) satisfying $\forall a, b \in \mathfrak{A}$

$$
\|a b\| \leq\|a\|\|b\| .
$$

If $\mathfrak{A}$ has an identity $e$, then we require that $\|e\|=1$.

Although we will denote the multiplication in an abstract Banach algebra simply by juxtaposition, the most important example of a Banach algebra multiplication in abstract harmonic analysis is convolution (see Section 1.5), which is denoted by $*$.

$C_{b}(T)$ and $C_{0}(T)$ are (commutative) Banach algebras with pointwise multiplication. Matrix algebras are noncommutative Banach algebras.

Many Banach algebras lack an identity (for example, $C_{0}(T)$ has an identity iff $T$ is compact). However, they may have something almost as good: 
Definition 1.1.3. $A$ bounded left approximate identity (BLAI) for a Banach algebra $\mathfrak{A}$ is a bounded net $\left(e_{\alpha}\right)$ in $\mathfrak{A}$ s.t. $\forall a \in \mathfrak{A}$

$$
\lim _{\alpha} e_{\alpha} a=a
$$

$A$ bounded right approximate identity (BRAI) is defined similarly. A net which is both a BLAI and a BRAI is called a bounded approximate identity (BAI).

Example. Let $G$ be a locally compact group. Then $L_{1}(G)$ has a standard BAI (with each element) of norm 1, see Proposition 2.42 in [14] for the construction.

Definition 1.1.4. Let $\mathfrak{A}$ be a Banach algebra. A left Banach $\mathfrak{A}$-module is a Banach space $E$ with a module map

$$
\cdot: \mathfrak{A} \times E \rightarrow E, \quad(a, x) \mapsto a \cdot x
$$

satisfying $\forall a \in \mathfrak{A}, x \in E$

$$
\|a \cdot x\| \leq C\|a\|\|x\|
$$

for some $C>0$. A right Banach module is defined similarly. A Banach $\mathfrak{A}$-bimodule is both a left and right Banach $\mathfrak{A}$-module s.t. the left and right actions commute.

Any Banach algebra is a module over itself.

If $\mathfrak{A}$ has an identity then we can factor $E=\mathfrak{A} \cdot E$ for any left $\mathfrak{A}$-module $E$. Surprisingly this is still possible if $\mathfrak{A}$ only has a BLAI, thanks to a result of the great Paul Cohen (who also proved that the Axiom of Choice and the Continuum Hypothesis are independent of ZF). 
Theorem 1.1.5 (Cohen's Factorization Theorem). Let $\mathfrak{A}$ be a Banach algebra with a BLAI bounded by $M, E$ be a left Banach $\mathfrak{A}-$ module, $x \in \overline{\mathfrak{A} \cdot E}$, and $\varepsilon>0$. Then $\exists a \in \mathfrak{A}$ and $y \in \mathfrak{A} \cdot E$ s.t.

- $\|a\| \leq M$.

- $\|y-x\|<\varepsilon$.

- $x=a \cdot y$.

A similar statement holds for right Banach $\mathfrak{A}$-modules over algebras $\mathfrak{A}$ with BRAIs.

Corollary 1.1.6. Let $\mathfrak{A}$ be a Banach algebra with a BLAI (respectively BRAI) and E be a left (resp. right) Banach $\mathfrak{A}-$ module. Then

$$
\overline{\mathfrak{A} \cdot E}=\mathfrak{A} \cdot E \quad(\text { resp. } \overline{E \cdot \mathfrak{A}}=E \cdot \mathfrak{A}) .
$$

\subsection{Linear operators}

Definition 1.2.1. Let $X$ and $Y$ be Banach spaces. A linear operator $T: X \rightarrow Y$ is bounded if it maps $X_{1}$ to a bounded set in $Y$. The vector space of all bounded linear operators $X \rightarrow Y$ is denoted by $B(X, Y)$ and $B(X):=B(X, X)$.

A linear operator is bounded iff it is continuous.

Let $X$ and $Y$ be a Banach space. Then $B(X, Y)$ is a Banach space with the norm

$$
\|T\|:=\sup _{x \in X_{1}}\|T x\|
$$

and $B(X)$ is a Banach algebra under composition.

A linear operator $T \in B(X, Y)$ s.t. $\|T x\|=\|x\| \quad \forall x \in X$ is called an isometry. 
It will be convenient to have a notation for the operators which commute with a module action.

Definition 1.2.2. Let $E$ be a (WLOG left) Banach $\mathfrak{A}$-module. Define

$$
B_{\mathfrak{A}}(E):=\{T \in B(E) \mid T(a \cdot x)=a \cdot T x \quad \forall a \in \mathfrak{A}, x \in E\} .
$$

We now define some important subspaces of $B(X, Y)$.

Definition 1.2.3. Let $X$ and $Y$ be Banach spaces.

(i) The finite-rank operators are

$$
\mathcal{F}(X, Y):=\{F \in B(X, Y) \mid \operatorname{dim} F(X)<\infty\}
$$

(ii) The approximable operators are

$$
\mathcal{A}(X, Y):=\overline{\mathcal{F}(X, Y)}
$$

(iii) The compact operators are

$$
\mathcal{K}(X, Y):=\left\{K \in B(X, Y) \mid \overline{K\left(X_{1}\right)} \subset Y \text { is compact }\right\}
$$

$\mathcal{A}(X, Y)$ and $\mathcal{K}(X, Y)$ are closed subspaces of $B(X, Y)$ and we have

$$
\mathcal{F}(X, Y) \subseteq \mathcal{A}(X, Y) \subseteq \mathcal{K}(X, Y) \subseteq B(X, Y)
$$

In general all of these inclusions are strict, and $\mathcal{F}(X):=\mathcal{F}(X, X), \mathcal{A}(X):=\mathcal{A}(X, X)$, and $\mathcal{K}(X):=\mathcal{K}(X, X)$ are (two-sided) ideals in $B(X)$. 


\subsection{Dual spaces}

We now introduce one of the most important concepts in functional analysis.

Definition 1.3.1. The (continuous) dual (space) of a Banach space $X$ is

$$
X^{*}:=B(X, \mathbb{C})
$$

The elements of $X^{*}$ are called (bounded linear) functionals (on $X$ ).

If $X$ is a Banach space, then for $f \in X^{*}$ and $x \in X$ we write

$$
\langle f, x\rangle:=f(x)
$$

Definition 1.3.2. If $X=Y^{*}$ for some Banach space $Y$, then $X$ is called a dual space and $Y$ is called a predual of $X$. Preduals are not (in general) unique, but we will sometimes distinguish a predual $Y$ of a dual space $X$ by writing $X_{*}=Y$.

Duals and preduals separate the points of a Banach space. We also have (thanks to Zorn's Lemma) the following result, one of the cornerstones of functional analysis.

Theorem 1.3.3 (Hahn-Banach). Let $Y$ be a subspace of a Banach space $X$ and $f \in Y^{*}$. Then $\exists \tilde{f} \in X^{*}$ s.t. $\left.\tilde{f}\right|_{Y}=f$ and $\|\tilde{f}\|=\|f\|$.

The concept of duality extends to linear maps:

Definition 1.3.4. Let $X$ and $Y$ be Banach spaces and $T \in B(X, Y)$. The adjoint of $T$ is the operator $T^{*} \in B\left(Y^{*}, X^{*}\right)$ defined by

$$
\left\langle T^{*} f, x\right\rangle:=\langle f, T x\rangle
$$

for $f \in Y^{*}$ and $x \in X$. 
The adjoint map * $: B(X, Y) \rightarrow B\left(Y^{*}, X^{*}\right)$ is isometric and adjoints of injections are surjections and vice versa.

We will use the following definitions:

Definition 1.3.5. Let $U \subseteq X$ a Banach space and $V \subseteq Y$ a dual space. The annihilator of $U$ is

$$
U^{\perp}:=\left\{f \in X^{*}:\langle f, u\rangle=0 \quad \forall u \in U\right\}
$$

a closed subspace of $X^{*}$. The preannihilator of $V$ is

$$
V_{\perp}:=\left\{h \in Y_{*}:\langle v, h\rangle=0 \quad \forall v \in V\right\}
$$

a closed subspace of $Y_{*}$.

\subsection{Banach space topologies}

The norm topology on a Banach space (arising from the metric $d(x, y)=\|x-y\|$ ) can be too strong (for example, the unit ball of any infinite dimensional Banach space is not compact). Thankfully, we have other topologies at our disposal.

There is an extensive theory of topological vector spaces (vector spaces endowed with a topology for which addition and scalar multiplication are continuous, see [34]). We only define three more, one on dual spaces and two on spaces of operators.

We will not rigorously define these topologies, only characterize their convergent nets (this is in fact sufficient, but would require a little work, see the section "Convergence Classes" in Chapter 2 of [23]). They are all Hausdorff and are generated by families of seminorms. 
Definition 1.4.1. Let $X, Y$ be a Banach spaces and $Z$ be a dual space.

The Strong Operator Topology on $B(X, Y)$ is determined by

$$
T_{\alpha} \stackrel{\text { SOT }}{\longrightarrow} T \quad \text { iff } \quad T_{\alpha} x \rightarrow T x \text { (in norm) } \quad \forall x \in X .
$$

The Weak Operator Topology on $B(X, Y)$ is determined by

$$
S_{\alpha} \stackrel{\text { WOT }}{\longrightarrow} S \quad \text { iff } \quad\left\langle f, S_{\alpha} x\right\rangle \rightarrow\langle f, S x\rangle \quad \forall x \in X, f \in Y^{*}
$$

The weak* topology on $Z$ is determined by

$$
f_{\alpha} \stackrel{w^{*}}{\rightarrow} f \quad \text { iff } \quad\left\langle f_{\alpha}, x\right\rangle \rightarrow\langle f, x\rangle \quad \forall x \in Z_{*} .
$$

It is clear that for nets $\left(T_{\alpha}\right)$ in $B(X, Y)$,

$$
T_{\alpha} \rightarrow T \text { (in norm) } \Rightarrow T_{\alpha} \stackrel{S O T}{\longrightarrow} T \quad \Rightarrow \quad T_{\alpha} \stackrel{\text { WOT }}{\longrightarrow} T .
$$

It is less clear that for bounded nets $\left(T_{\alpha}\right)$ in $B(X, Y)$,

$$
T_{\alpha} \stackrel{W O T}{\longrightarrow} T \quad \Longleftrightarrow \quad T_{\alpha} \stackrel{w^{*}}{\rightarrow} T .
$$

It is not even clear that $B(X, Y)$ supports a weak* topology, since we do not know $a$ priori that $B(X, Y)$ is a dual space. We will address this issue in Chapter 3 where we will see that $B(X, Y)$ is a dual space for all Banach spaces $X$ and $Y$.

The weak* topology, in particular, is incredibly useful, due mainly to the following result, one of the most important in functional analysis.

Theorem 1.4.2 (Banach-Alaoglu). The unit ball of a dual space is $w^{*}$-compact. 
Bounded linear operators between dual spaces are not in general $w^{*}-w^{*}$-continuous. Those that are deserve a special name.

Definition 1.4.3. Let $X$ and $Y$ be dual Banach spaces. Let

$$
B^{\sigma}(X, Y):=\left\{T \in B(X, Y) \mid T \text { is } w^{*}-w^{*} \text {-continuous }\right\}
$$

The elements of $B^{\sigma}(X, Y)$ are called normal operators.

It is easy to see that adjoint operators (Definition 1.3.4) are normal. The converse is also true:

Proposition 1.4.4. If $T \in B\left(Y^{*}, X^{*}\right)$ is normal (i.e. $w^{*}$-w*-continuous), then $T=S^{*}$ for some $S \in B(X, Y)$.

\subsection{Abstract harmonic analysis}

Definition 1.5.1. A topological group $G$ is a group and a topological space such that the map

$$
G \times G \rightarrow G, \quad(g, h) \mapsto g h^{-1}
$$

is continuous (where $G \times G$ is endowed with the product topology). A locally compact group is a topological group which is a locally compact Hausdorff space.

Examples. Any discrete or compact group is locally compact.

Locally compact groups are the basic objects of abstract harmonic analysis. They support a rich theory because of the following definition and result.

Definition 1.5.2. Let $G$ be a locally compact group. A regular Borel measure $\lambda$ on 
$G$ is a left Haar measure if $\forall t \in G$ and $A \in \mathscr{B}(G)$ (the Borel $\sigma$-algebra on $G$ )

$$
\mu(t A)=\mu(A)
$$

and $a$ right Haar measure if $\forall t \in G$ and $A \in \mathscr{B}(G)$

$$
\mu(A t)=\mu(A)
$$

Theorem 1.5.3. Let $G$ be any locally compact group. Then there exist unique up to positive scalar multiple left and right Haar measures $\lambda$ and $\rho$ on $G$. Furthermore, there is a homomorphism $\Delta: G \rightarrow \mathbb{R}^{+}$s.t. $\forall t \in G$ and $A \in \mathscr{B}(G)$

$$
\lambda(A t)=\Delta(t) \lambda(A) \quad \text { and } \quad \rho(t A)=\Delta(t)^{-1} \rho(A)
$$

$\Delta$ is called the modular function of $G$. Groups for which the modular function is constantly 1 are called unimodular. Abelian, discrete, and compact groups are unimodular.

We fix a left Haar measure $\lambda$ on $(G, \mathscr{B}(G))$ and write $L_{p}(G)=L_{p}(\lambda)$ and $d s=d \lambda(s)$. For any locally compact group $G, L_{1}(G)$ is a Banach algebra with convolution product $*$ defined by

$$
f * g(x)=\int_{G} f(y) g\left(y^{-1} x\right) d y
$$

for $f, g \in L_{1}(G)$.

Given a locally compact group $G$, the set of all complex (and hence finite) regular Borel measures on $G$ is a Banach space with the total variation norm 


$$
\|\mu\|:=|\mu(G)|
$$

for $\mu \in M(G)$. By the Riesz Representation Theorem,

$$
M(G)=C_{0}(G)^{*}
$$

via the duality

$$
\langle\mu, f\rangle=\int_{G} f d \mu
$$

for $\mu \in M(G)$ and $f \in C_{0}(G) . M(G)$ is moreover a Banach algebra with the convolution product defined via duality with $C_{0}(G)$ :

$$
\int_{G} f d(\mu * \nu)=\langle\mu * \nu, f\rangle:=\int_{G} \int_{G} f(x y) d \mu(x) d \nu(y)
$$

for $\mu, \nu \in M(G)$ and $f \in C_{0}(G) . M(G)$ is called the measure algebra of $G$.

The group algebra $L_{1}(G)$ is identified with a closed subalgebra of $M(G)$ by integrating over Borel subsets of $G$ :

$$
\begin{gathered}
L_{1}(G) \hookrightarrow M(G), \quad f \mapsto \mu_{f} \\
\mu_{f}(A):=\int_{A} f
\end{gathered}
$$

for $A \in \mathscr{B}(G)$.

If $G$ is discrete then $M(G)=\ell_{1}(G)$ and $M(G)$ is commutative iff $L_{1}(G)$ is commutative iff $G$ is abelian.

We liberally use Fubini's Theorem adapted for abstract harmonic analysis (see Sections 12 and 13 of [20] and the discussion on p.88 of [30]). 
Theorem 1.5.4 (Fubini). Let $G$ be a locally compact group, $\mu, \nu \in M(G)$, and $f \in L_{1}(\mu \times \nu)$. Then

$$
\int_{G \times G} f d(\mu \times \nu)=\int_{G} \int_{G} f d \mu d \nu=\int_{G} \int_{G} f d \nu d \mu .
$$

Let $G$ be a topological group. The left uniformly continuous functions on $G$ are

$$
\operatorname{LUC}(G):=\left\{f \in C_{b}(G) \mid G \ni t \mapsto l_{t} f \text { is }\|\cdot\|_{\infty} \text {-continuous }\right\}
$$

$\operatorname{RUC}(G)$ (the right uniformly continuous functions) is defined similarly and

$$
\operatorname{RUC}(G)=\overline{\operatorname{LUC}(G)}
$$

$\operatorname{LUC}(G)$ and $\operatorname{RUC}(G)$ are closed subalgebras of $C_{b}(G)$ which contain $C_{0}(G)$.

If $G$ is a locally compact group, then $M(G) \subset \operatorname{LUC}(G)^{*}$ via the duality

$$
\langle\mu, f\rangle:=\int_{G} f d \mu
$$

for $f \in \operatorname{LUC}(G)$ and $\mu \in M(G)$. In fact, by Bemerkung 2.3.2 in [30], $M(G)$ is $w^{*}$-dense in $\operatorname{LUC}(G)^{*}$, i.e.

$$
\overline{M(G)}^{\omega^{*}}=\operatorname{LUC}(G)^{*}
$$




\section{Chapter 2}

\section{Second Duals of Banach Algebras}

Here we define the Arens products on the second duals of Banach algebras, two products which extend the product on the original algebra, and related concepts, some of which have yet to be defined in the literature. Arens products date back to 1951, but there has been a recent resurgence of interest in them and many good results are very new or still in the pipeline - it seems that only now are mathematicians ready for them.

The theory is almost peculiar to general Banach algebra theory and abstract harmonic analysis: operator algebras are uninteresting from this point of view (see the example after Definition 2.3.3). If nothing else, this chapter is intended to convince the reader of how much is lost when one restricts one's attention to reflexive (and especially Hilbert) spaces or operator algebras. 


\section{$2.1 \quad$ Second duals}

The second dual (or bidual) of a Banach space $X$ is $X^{* *}:=\left(X^{*}\right)^{*}$.

Every Banach space embeds isometrically into its second dual via the map which sends a point to the evaluation functional at that point, i.e.

$$
\begin{gathered}
X \ni x \mapsto \hat{x} \in X^{* *} \\
\langle\hat{x}, f\rangle:=\langle f, x\rangle
\end{gathered}
$$

for $f \in X^{*}$. Thus we identify $X$ with a closed subspace of $X^{* *}$.

Spaces for which this map is surjective (and hence an isomorphism) are called reflexive. Hilbert (and hence finite-dimensional) spaces are reflexive (in fact selfdual). For $p \in(1, \infty), L_{p}$ spaces are reflexive since $L_{p}^{*}=L_{q}$.

However, from our point of view, reflexive spaces are boring. But even for nonreflexive spaces, a lot can be said about this embedding.

Theorem 2.1.1 (Goldstine). Let $X$ be a Banach space. Then the unit ball of $X$ is $w^{*}$-dense in the unit ball of $X^{* *}$, i.e.

$$
{\overline{X_{1}}}^{{ }^{*}}=\left(X^{* *}\right)_{1}
$$

Corollary 2.1.2. Every Banach space $X$ is $w^{*}$-dense in its second dual, i.e.

$$
\bar{X}^{w^{*}}=X^{* *}
$$

Proposition 2.1.3. Let $X$ be a Banach space and $m \in X^{* *}$ be $w^{*}$-continuous.

Then $m \in X$. Using the notation of Definition 1.4.3, $B^{\sigma}\left(X^{* *}, \mathbb{C}\right)=X$. 
A function is continuous iff it commutes with limits of nets, and often there is a bound on the cardinality of nets we need consider (of course, the cardinality of the topology on the domain is always sufficient, but we may be able to do much better). For example, on first countable spaces, nets of cardinality $\aleph_{0}$ (i.e. sequences) are sufficient. In our context and in view of Proposition 2.1.3, this idea is captured by the following definitions.

Definition 2.1.4. A linear functional $f \in X^{*}$ is $w^{*}$ - $\Omega$-continuous for a cardinal $\Omega$ if for every convergent net $\left(x_{\alpha}\right)_{\alpha \in I}$ with $|I| \leq \Omega$,

$$
\left\langle f, w^{*}-\lim _{\alpha} x_{\alpha}\right\rangle=\lim _{\alpha}\left\langle f, x_{\alpha}\right\rangle .
$$

Definition 2.1.5. A Banach space $X$ has Mazur's proprty of level $\Omega$ a cardinal if every $m \in X^{* *}$ which is $w^{*}-\Omega$-continuous is in fact in $X$. A space is said to have Mazur's property if it has Mazur's property of level $\aleph_{0}$.

Reflexive spaces trivially have Mazur's property. Separable spaces have Mazur's property. $L_{1}(G)$ has Mazur's property of level $\kappa(G)$, the compact covering number of $G$ (the least cardinality of a covering of $G$ by compacta).

Usually two is the magic number for duals, however there is at least one important result for triple duals.

Proposition 2.1.6 (The Dixmier Projection). Let $X$ be a Banach space. Then

$$
X^{* * *}=X^{*} \oplus X^{\perp} .
$$

We end this section with two vector-valued integrals, both "pointwise" using linear functionals. This allows us to integrate in Banach spaces using (usual) scalar measures. They are included here since the second involves second duals. 
Definition 2.1.7. Let $(M, \mu)$ be a complex measure space and $X$ be a Banach space.

(i) A bounded function $f: M \rightarrow X^{*}$ is called weak ${ }^{*}$ integrable if the function

$$
M \rightarrow \mathbb{C}, \quad t \mapsto\langle f(t), x\rangle
$$

is integrable $\forall x \in X$ and we define the functional $\int_{M}^{w^{*}} f d \mu \in X^{*}$ by

$$
\left\langle\int_{M}^{w^{*}} f d \mu, x\right\rangle:=\int_{M}\langle f(t), x\rangle d \mu(t)
$$

$\int_{M} f d \mu$ is called the weak* integral of $f$ w.r.t. $\mu$.

(ii) $A$ bounded function $f: M \rightarrow X$ is called weakly (or Pettis) integrable if the function

$$
M \rightarrow \mathbb{C}, \quad t \mapsto\langle h, f(t)\rangle
$$

is integrable $\forall h \in X^{*}$ and the functional $\int_{M}^{w} f d \mu \in X^{* *}$ defined by

$$
\left\langle\int_{M}^{w} f d \mu, h\right\rangle:=\int_{M}\langle h, f(t)\rangle d \mu(t)
$$

lies in $X . \int_{M}^{w} f d \mu \in X$ is called the weak (or Pettis) integral of $f$ w.r.t $\mu$.

The functionals $\int_{M}^{w^{*}} f d \mu$ and $\int_{M}^{w} f d \mu$ are clearly linear and indeed bounded, since $\forall x \in X, h \in X^{*}$,

$$
\begin{aligned}
& \left|\left\langle\int_{M}^{w^{*}} f d \mu, x\right\rangle\right|=\int_{M}|\langle f(t), x\rangle| d|\mu|(t) \leq \sup _{t \in M}\|f(t)\|\|x\||\mu|(M) \\
& \left|\left\langle h, \int_{M}^{w} f d \mu\right\rangle\right|=\int_{M}|\langle h, f(t)\rangle| d|\mu|(t) \leq\|h\| \sup _{t \in M}\|f(t)\||\mu|(M) .
\end{aligned}
$$


Thanks to the embedding $X \hookrightarrow X^{* *}$, if $X$ is a dual space then the weak* integral coincides with the weak integral when the latter exists.

It should be noted that in the definition of the weak integral we require the linear functional we defined in $X^{* *}$ to be in $X$. This can be difficult to check, but we have the following handy sufficient condition, a special case of Theorem A3.3 in [14].

Proposition 2.1.8. Let $X$ be a Banach space, $G$ be a locally compact group and $\mu \in M(G)$. Then if $f: G \rightarrow X$ is bounded and continuous, then the weak integral $\int_{G}^{w} f d \mu$ exists.

\subsection{Arens products}

Since a Banach algebra $\mathfrak{A}$ embeds isometrically into $\mathfrak{A}^{* *}$, we can (and do) ask if it is possible to extend the multiplication on $\mathfrak{A}$ to $\mathfrak{A}^{* *}$. Richard Arens showed in 1951 in [1] and [2] that this can be done in two canonical ways, yielding what are now called the Arens products.

Let $m, n \in \mathfrak{A}^{* *}$. By the Corollary to Goldstine's Theorem (2.1.2), $\exists$ nets $\left(a_{\alpha}\right)$ and $\left(b_{\beta}\right)$ in $\mathfrak{A}$ with

$$
m=w^{*}-\lim _{\alpha} a_{\alpha} \quad \text { and } \quad n=w^{*}-\lim _{\beta} b_{\beta}
$$

So we could attempt to multiply $m$ and $n$ by defining

$$
m n=\left(w^{*}-\lim _{\alpha} a_{\alpha}\right)\left(w^{*}-\lim _{\beta} b_{\beta}\right):=w^{*}-\lim _{\alpha} w^{*}-\lim _{\beta} a_{\alpha} b_{\beta}
$$

but we could just have easily taken the other iterated limit and defined

$$
m n=\left(w^{*}-\lim _{\alpha} a_{\alpha}\right)\left(w^{*}-\lim _{\beta} b_{\beta}\right):=w^{*}-\lim _{\beta} w^{*}-\lim _{\alpha} a_{\alpha} b_{\beta}
$$


and it is not obvious that the iterated limits exist, that this "product" is a Banach algebra product, or even if it is well-defined - to quote Neufang, "like women, the topological viewpoint is beautiful, but difficult to understand".

We will define two products on $\mathfrak{A}^{* *}$ algebraically using module actions.

Definition 2.2.1. Let $m, n \in \mathfrak{A}^{* *}, f \in \mathfrak{A}^{*}$, and $a, b \in \mathfrak{A}$. The first (or left) Arens product, denoted $\square$, is defined by the cascading definitions

$$
\begin{aligned}
\langle m \square n, f\rangle & :=\langle m, n \square f\rangle \\
\langle n \square f, a\rangle & :=\langle n, f \square a\rangle \\
\langle f \square a, b\rangle & :=\langle f, a b\rangle .
\end{aligned}
$$

The second (or right) Arens product, denoted $\diamond$, is defined oppositely:

$$
\begin{gathered}
\langle m \diamond n, f\rangle:=\langle n, f \diamond m\rangle \\
\langle f \diamond m, a\rangle:=\langle m, a \diamond f\rangle \\
\langle a \diamond f, b\rangle:=\langle f, b a\rangle .
\end{gathered}
$$

and $\diamond$ are associative bilinear products on $\mathfrak{A}^{* *}$ which make $\mathfrak{A}^{* *}$ a Banach algebra and extend the product on $\mathfrak{A}$. If $m=w^{*}-\lim _{\alpha} a_{\alpha}$ and $n=w^{*}-\lim _{\beta} b_{\beta}$ with the $a_{\alpha}, b_{\beta} \in \mathfrak{A}$, then

$$
\begin{aligned}
& m \square n=w^{*}-\lim _{\alpha} w^{*}-\lim _{\beta} a_{\alpha} b_{\beta} \\
& m \diamond n=w^{*}-\lim _{\beta} w^{*}-\lim _{\alpha} a_{\alpha} b_{\beta} .
\end{aligned}
$$

Furthermore, $\square$ makes $\mathfrak{A}^{*}$ a right $\mathfrak{A}$-module and left $\mathfrak{A}^{* *}$-module and $\diamond$ makes $\mathfrak{A}^{*}$ a left $\mathfrak{A}$-module and right $\mathfrak{A}^{* *}$-module. We use the same symbol for three different module actions, but no confusion will arise. 
Remark 2.2.2. If $\mathfrak{A}$ is commutative then $\forall m, n \in \mathfrak{A}^{* *}$

$$
m \square n=n \diamond m .
$$

\subsection{Topological centres and DTC sets}

We would like to know when the two Arens products coincide. We measure their agreement via the following definitions.

Definition 2.3.1. Let $\mathfrak{A}$ be a Banach algebra with Arens products $\square$ and $\diamond$. The left (or first) and right (or second) topological centre of $\mathfrak{A}^{* *}$ are respectively

$$
\begin{aligned}
\mathcal{Z}_{t}^{1}\left(\mathfrak{A}^{* *}\right): & =\left\{m \in \mathfrak{A}^{* *} \mid m \square n=m \diamond n \quad \forall n \in \mathfrak{A}^{* *}\right\} \\
& =\left\{m \in \mathfrak{A}^{* *} \mid \text { the map } \mathfrak{A}^{* *} \ni n \mapsto m \square n \text { is } w^{*}-w^{*} \text {-continuous }\right\} \\
\mathcal{Z}_{t}^{2}\left(\mathfrak{A}^{* *}\right) & :=\left\{n \in \mathfrak{A}^{* *} \mid m \square n=m \diamond n \quad \forall m \in \mathfrak{A}^{* *}\right\} \\
& =\left\{n \in \mathfrak{A}^{* *} \mid \text { the map } \mathfrak{A}^{* *} \ni m \mapsto m \diamond n \text { is } w^{*}-w^{*} \text {-continuous }\right\} .
\end{aligned}
$$

Both centres are (norm-)closed subalgebras of $\mathfrak{A}^{* *}$ for either Arens product and

$$
\mathfrak{A} \subseteq \mathcal{Z}_{t}^{1}\left(\mathfrak{A}^{* *}\right) \cap \mathcal{Z}_{t}^{2}\left(\mathfrak{A}^{* *}\right)
$$

Remark 2.3.2. If $\mathfrak{A}$ is commutative, then by Remark 2.2.2,

$$
\mathcal{Z}_{t}^{1}\left(\mathfrak{A}^{* *}\right)=\mathcal{Z}_{t}^{2}\left(\mathfrak{A}^{* *}\right)=\mathcal{Z}\left(\mathfrak{A}^{* *}, \square\right)=\mathcal{Z}\left(\mathfrak{A}^{* *}, \diamond\right)
$$

where $\mathcal{Z}$ denotes the algebraic centre.

The reader may note that, using the second definition of the topological centres, 
two additional topological centres appear to be missing, namely

$$
\begin{aligned}
& \mathcal{Z}_{t}^{3 ?}\left(\mathfrak{A}^{* *}\right):=\left\{m \in \mathfrak{A}^{* *} \mid \text { the map } \mathfrak{A}^{* *} \ni n \mapsto m \square n \text { is } w^{*} \text {-w } w^{*} \text {-continuous }\right\} \\
& \mathcal{Z}_{t}^{4 ?}\left(\mathfrak{A}^{* *}\right):=\left\{n \in \mathfrak{A}^{* *} \mid \text { the map } \mathfrak{A}^{* *} \ni m \mapsto m \diamond n \text { is } w^{*}-w^{*} \text {-continuous }\right\}
\end{aligned}
$$

However, both of these extra centres are always equal to the entire $\mathfrak{A}^{* *}$.

The topological centres measure to what extent the two Arens products coincide. This naturally leads to the following classification of Banach algebras.

Definition 2.3.3 (2.18 in [10]). A Banach algebra $\mathfrak{A}$ is Arens regular (AR) if

$$
\mathcal{Z}_{t}^{1}\left(\mathfrak{A}^{* *}\right)=\mathcal{Z}_{t}^{2}\left(\mathfrak{A}^{* *}\right)=\mathfrak{A}^{* *}
$$

left strongly Arens irregular (LSAI) if

$$
\mathcal{Z}_{t}^{1}\left(\mathfrak{A}^{* *}\right)=\mathfrak{A},
$$

right strongly Arens irregular (RSAI) if

$$
\mathcal{Z}_{t}^{2}\left(\mathfrak{A}^{* *}\right)=\mathfrak{A},
$$

and strongly Arens irregular (SAI) if it is LSAI and RSAI.

Examples. Any closed subalgebra of $B(\mathcal{H})$ for some Hilbert space $\mathcal{H}$ (in particular, any $\mathrm{C}^{*}$-algebra) is Arens regular (Theorem 7.1 in $[6]$ ) but $L_{1}(G)$ is strongly Arens irregular for every locally compact group $G$ (Theorem 1 in [25]). Note that this implies that $L_{1}(G)$ can never embed isometrically into any $B(\mathcal{H})$.

Note that with these definitions, a Banach algebra can be both Arens regular and strongly Arens irregular, this is the case iff $\mathfrak{A}$ is reflexive. 
Remark 2.3.4. We do not define left and right variants of Arens regularity due to the simple observation that

$\mathfrak{A}$ is Arens regular $\quad \Longleftrightarrow \quad \mathcal{Z}_{t}^{1}\left(\mathfrak{A}^{* *}\right)=\mathfrak{A}^{* *} \quad \Longleftrightarrow \quad \mathcal{Z}_{t}^{2}\left(\mathfrak{A}^{* *}\right)=\mathfrak{A}^{* *}$.

However, the analogous statement for strong Arens irregularity (i.e., replacing $\mathfrak{A}^{* *}$ on the right side of the above equalities with $\mathfrak{A}$ ) is not true. The first counterexample was given by Neufang in [28]. This algebra is the main object of study of this thesis, we will study it in Chapter 4 and give new results for it in Chapter 6.

The topological centre conditions are indeed hefty requirements, one desires weaker sufficient conditions for elements of the second dual to be in the centres. Surprisingly it is sometimes enough to test against small finite sets, motivating the following definitions.

Definition 2.3.5. A set $\mathfrak{D} \subsetneq \mathfrak{A}^{* *}$ is a left DTC set for $\mathfrak{A}$ if for $m \in \mathfrak{A}^{* *}$

$$
m \square d=m \diamond d \quad \forall d \in \mathfrak{D} \Rightarrow m \in \mathcal{Z}_{t}^{1}\left(\mathfrak{A}^{* *}\right)
$$

or equivalently if

$$
m \square w^{*}-\lim _{\alpha} d_{\alpha}=w^{*}-\lim _{\alpha} m \square d_{\alpha}
$$

for every net $\left(d_{\alpha}\right)$ in $\mathfrak{A}^{* *}$ converging $w^{*}$ to some $d \in \mathfrak{D}$ implies that $m \in \mathcal{Z}_{t}^{1}\left(\mathfrak{A}^{* *}\right)$. Right DTC sets are defined similarly. A DTC set is a left and right DTC set.

We could have defined (left/right) DTC sets for $\mathfrak{A}$ to be subsets of $\mathfrak{A}^{* *} \backslash \mathfrak{A}$, since if $\mathfrak{D}$ is a (left/right) DTC set for $\mathfrak{A}$ then so is $\mathfrak{D} \backslash \mathfrak{A}$. The reason we do not is because it can be very difficult to tell when $m \in \mathfrak{A}^{* *}$ is in fact in $\mathfrak{A}$.

DTC sets have to somehow encode all the topological and algebraic information 
about the multiplication, so small DTC sets are hard to come by. One of the few algebras known to have a small DTC set is For infinite discrete groups $G, \ell_{1}(G)$ has a 2-point left DTC set which is obtained by interpreting Theorem 12.22 in [11] in the case that $G$ is discrete. This result can be reflected to yield a 2-point right DTC set.

We introduce the following terminology for convenience.

Definition 2.3.6. A Banach algebra $\mathfrak{A}$ is $n$-LDTC/n-RDTC/n-DTC, $n \in \mathbb{N}$, if there is a n-point left $D T C /$ right $D T C / D T C$ set for $\mathfrak{A}^{* *}$.

Note that if $\mathfrak{A}$ has $n$-point left and right DTC sets, then by this definition $\mathfrak{A}$ is not necessarily $n$-DTC, for this they must be the same $n$ points.

Remark 2.3.7. $\mathfrak{A}$ is $0-(\mathrm{L} / \mathrm{R}) \mathrm{DTC}$ iff $\mathfrak{A}$ is $\mathrm{AR}$.

Remark 2.3.8. No commutative Arens irregular Banach algebra can be 1-DTC, since (by Remark 2.2.2) $\forall a \in \mathfrak{A}^{* *} \backslash \mathcal{Z}\left(\mathfrak{A}^{* *}\right)$

$$
a \square a=a \diamond a
$$

which would imply (by Remark 2.3.2) that $a \in \mathcal{Z}\left(\mathfrak{A}^{* *}\right)$.

However, $\ell_{1}\left(\mathbb{F}_{2}\right)$ is 1-LDTC, see Example 12.21 in [11]. Recall that $\mathbb{F}_{2}$ is the free group on 2 generators (with the discrete topology). 


\section{Chapter 3}

\section{Tensor Products of Banach Spaces}

There is a beautiful theory of tensor products of Banach spaces pioneered by Alexander Grothendieck ([17]) of which we will barely scratch the surface. For the purposes of this thesis they are merely a tool, albeit an absolutely essential one. Tensor products allow us to realize the bounded linear operators on any Banach space as a dual space and motivates the definition of the nuclear operators, the object of study of this thesis.

There are many ways to norm the tensor product of Banach spaces, here we give only the two we need (not coincidentally, they are the most important). We then introduce two technical properties of Banach spaces which allow us to identify these tensor products with more familiar objects.

The reader is strongly encouraged to see Ryan's excellent book on tensor products of Banach spaces [33] for a better understanding of this material, it is truly something every analyst should know. 


\subsection{The algebraic tensor product}

Let $U$ and $V$ be vector spaces. We wish to define a vector space which is the "product" of $U$ and $V$ in the sense that bilinear maps on $U \times V$ can be identified with linear maps on this new space. We will call this space the tensor product of $U$ and $V$ and denote it by $U \otimes V$. There are many equivalent ways of defining the tensor product of two vector spaces, we proceed as in [33]. Denote the vector space of all bilinear maps $U \times V \rightarrow \mathbb{C}$ by $\mathfrak{B}(U \times V)$. We define $U \otimes V$ to be a subspace of $\mathfrak{B}(U \times V)^{\sharp}$, the algebraic dual of $\mathfrak{B}(U \times V)$.

Definition 3.1.1. For $u \in U, v \in V$, the elementary tensor $u \otimes v \in \mathfrak{B}(U \times V)^{\sharp}$ is defined by

$$
\langle u \otimes v, T\rangle:=T(u, v) .
$$

for $T \in \mathfrak{B}(U \times V)$. The tensor product of $U$ and $V$ is

$$
U \otimes V:=\operatorname{span}\{u \otimes v \mid u \in U, v \in V\}
$$

a subspace of $\mathfrak{B}(U \times V)^{\sharp}$.

Obviously $U \otimes V \cong V \otimes U$ via a canonical map and it is clear that the map $\otimes: U \times V \rightarrow U \otimes V,(u, v) \mapsto u \otimes v$ is itself bilinear.

Any tensor $w \in U \otimes V$ can be expressed as a finite sum of elementary tensors, however such a representation is not unique. The least $n \in \mathbb{N}$ s.t. $w$ may be written as the sum of $n$ elementary tensors is called the rank of $v$. 
Proposition 3.1.2. If $\left\{u_{\alpha}\right\}_{\alpha \in A}$ and $\left\{v_{\beta}\right\}_{\beta \in B}$ are (Hamel) bases for vector spaces $U$ and $V$, respectively, then $\left\{u_{\alpha} \otimes v_{\beta}\right\}_{(\alpha, \beta) \in A \times B}$ is a basis for $U \otimes V$, and consequently

$$
V \otimes \mathbb{C} \cong \mathbb{C} \otimes V \cong V
$$

Now let $X$ and $Y$ be Banach spaces. We want $X \otimes Y$ to be a Banach space, so the first thing we need is a norm on $X \otimes Y$. It is natural to require that

$$
\|x \otimes y\| \leq\|x\|\|y\|
$$

(so that the map $\otimes$ is jointly continuous). Such a norm is called a cross norm.

In the following two sections, we will give two cross norms on the tensor product of Banach spaces and study the corresponding normed spaces.

\subsection{The injective tensor product}

We may identify tensors $\sum_{i=1}^{n} u_{i} \otimes v_{i} \in U \otimes V$ with linear maps $U^{\sharp} \rightarrow V$ by defining

$$
\left(\sum_{i=1}^{n} u_{i} \otimes v_{i}\right)(f):=\sum_{i=1}^{n}\left\langle f, u_{i}\right\rangle v_{i}
$$

for $f \in U^{\sharp}$. Similarly we may identify such tensors with linear maps $V^{\sharp} \rightarrow U$.

Now let $X$ and $Y$ be Banach spaces. Then we can identify tensors in $X \otimes Y$ with (finite rank, and hence bounded) linear maps $X^{*} \rightarrow Y$ and thus $X \otimes Y$ inherits a norm from $B\left(X^{*}, Y\right)$. This norm is denoted $\|\cdot\|_{\varepsilon}$ and is given explicitly by

$$
\|u\|_{\varepsilon}=\sup _{f \in X_{1}^{*}}\left\{\left\|\sum_{i=1}^{n}\left\langle f, x_{i}\right\rangle y_{i}\right\|: u=\sum_{i=1}^{n} x_{i} \otimes y_{i}\right\} .
$$


From this we can see that

$$
\|x \otimes y\|_{\varepsilon} \leq\|x\|\|y\|
$$

$\forall x \in X, y \in Y$. In fact, we always always have equality in the above expression (i.e. $\left.\|x \otimes y\|_{\varepsilon}=\|x\|\|y\|\right)$ by Proposition 3.1(b) in [33].

It is clear that since $\mathcal{F}\left(X^{*}, Y\right)$ is not closed in $B\left(X^{*}, Y\right),\left(X \otimes Y,\|\cdot\|_{\varepsilon}\right)$ is not complete. The completion of $\left(X \otimes Y,\|\cdot\|_{\varepsilon}\right)$ is denoted $X \otimes_{\varepsilon} Y$ and called the injective tensor product of $X$ and $Y$. Since $X \otimes Y \hookrightarrow B\left(X^{*}, Y\right)$,

$$
X \otimes_{\varepsilon} Y=\overline{X \otimes Y}^{B\left(X^{*}, Y\right)} .
$$

The elements of $X \otimes_{\varepsilon} Y$ are still referred to as tensors. Clearly $X \otimes_{\varepsilon} Y \simeq Y \otimes_{\varepsilon} X$.

If $Z=X^{*}$ is a dual space, we may instead regard $Z \otimes Y=X^{*} \otimes Y$ as a subspace of $B(X, Y)$ in the obvious way. It is clear that the operators obtained via this identification are exactly $\mathcal{F}(X, Y)$. So by uniqueness of the completion,

$$
X^{*} \otimes_{\varepsilon} Y=\overline{X \otimes Y}^{B(X, Y)}=\mathcal{A}(X, Y) .
$$

We would prefer to have equality with the compact operators, so we will give a partial answer to the question "When is every compact operator approximable?" in Section 3.4. 


\subsection{The projective tensor product}

We will need a second tensor product of Banach spaces. By subadditivity of the norm and cross norm inequality, we must have

$$
\left\|\sum_{i=1}^{n} x_{i} \otimes y_{i}\right\| \leq \sum_{i=1}^{n}\left\|x_{i}\right\|\left\|y_{i}\right\|
$$

and this must hold for all representations of $\sum_{i=1}^{n} x_{i} \otimes y_{i}$. So define the projective norm on $X \otimes Y$ by

$$
\|u\|_{\pi}:=\inf \left\{\sum_{i=1}^{n}\left\|x_{i}\right\|\left\|y_{i}\right\|: u=\sum_{i=1}^{n} x_{i} \otimes y_{i}, n \in \mathbb{N}\right\}
$$

The projective norm is indeed a norm and satisfies $\|x \otimes y\|_{\pi}=\|x\|\|y\|$ (Proposition 2.1 in [33]). It is clearly the largest possible cross norm on $X \otimes Y$.

Again $\left(X \otimes Y,\|\cdot\|_{\pi}\right)$ is not complete. The completion is denoted $X \otimes_{\pi} Y$ and called the projective tensor product of $X$ and $Y$. As usual, $X \otimes_{\pi} Y \simeq Y \otimes_{\pi} X$.

Elements of the injective tensor product can only be described as limits of sequences of finite rank operators. Elements of the projective tensor product, on the other hand, can be realized in a much more convenient way:

Proposition 3.3.1 (Proposition 2.8 in [33]). Let $u \in X \otimes_{\pi} Y$ and $\varepsilon>0$. Then there are sequences $\left(x_{n}\right)$ and $\left(y_{n}\right)$ in $X$ and $Y$ respectively s.t.

$$
u=\sum_{n=1}^{\infty} x_{n} \otimes y_{n}
$$

and

$$
\|u\|_{\pi}<\sum_{n=1}^{\infty}\left\|x_{n}\right\|\left\|y_{n}\right\|+\varepsilon
$$


It is immediate from this result that

$$
\|u\|_{\pi}=\inf \left\{\sum_{n=1}^{\infty}\left\|x_{n}\right\|\left\|y_{n}\right\|: u=\sum_{n=1}^{\infty} x_{n} \otimes y_{n}\right\}
$$

The following identification for duals of projective tensor products is extremely useful.

Proposition 3.3.2. Let $X$ and $Y$ be Banach spaces. Then

$$
\left(X \otimes_{\pi} Y\right)^{*}=B\left(X, Y^{*}\right)
$$

via the duality

$$
\left\langle T, \sum_{n=1}^{\infty} x_{n} \otimes y_{n}\right\rangle:=\sum_{n=1}^{\infty}\left\langle T x_{n}, y_{n}\right\rangle .
$$

for $T \in B\left(X, Y^{*}\right)$ and $\sum_{n=1}^{\infty} \in X \otimes_{\pi} Y \in X \otimes_{\pi} Y$.

See Section 2.2 of [33] for the proof.

As with the injective tensor product, we will now identify projective tensors with linear operators. However, since we completed $X \otimes Y$ before making this identification, the resulting operators are different. Define the map

$$
J: X^{*} \otimes_{\pi} Y \rightarrow B(X, Y)
$$

by for $\sum_{n=1}^{\infty} f_{n} \otimes y_{n} \in X^{*} \otimes_{\pi} Y$ and $x \in X$

$$
J\left(\sum_{n=1}^{\infty} f_{n} \otimes y_{n}\right)(x):=\sum_{n=1}^{\infty}\left\langle f_{n}, x\right\rangle y_{n}
$$

The resulting operators are called nuclear operators. We write

$$
\mathcal{N}(X, Y):=J\left(X^{*} \otimes_{\pi} Y\right)
$$


$J$ is clearly linear, but it is imperative to note that it may fail to be injective. So the most we can say is that (by the First Isomorphism Theorem),

$$
\mathcal{N}(X, Y) \cong\left(X^{*} \otimes_{\pi} Y\right) / \operatorname{ker} J
$$

We will give a sufficient condition for $J$ to be injective in the following section.

In general, the nuclear operators are distinct from the more familiar classes of approximable, compact, and bounded operators. However, since each nuclear operator is a series of rank-one operators, they are approximable and hence also compact. The nuclear operators on $L_{p}(G)$ will be studied more thoroughly in Chapter 4.

\subsection{The Approximation and Radon-Nikodym Properties}

Here we define two technical properties of Banach spaces, one to answer the questions we asked above about tensor products, and the other to make an identification for the dual space of the injective tensor product.

Definition 3.4.1. A Banach space $X$ has the Approximation Property (AP) if for every compact $K \subset X$ and $\varepsilon>0$ there is a finite-rank operator $S \in \mathcal{F}(X)$ s.t. $\|x-S x\|<\varepsilon \quad \forall x \in K$

Examples. References are to [33]

(i) (Example 4.2) $C(K)$ has AP for every compact space $K$.

(ii) (Example 4.3) Every Banach space with a Schauder basis has AP.

(iii) (Example 4.5) $L_{p}(\mu)$ has AP for every measure $\mu$ and $p \in[1, \infty)$. 
Not every space has AP, but this is far from obvious. The first example of a space which fails to have AP was $B\left(\ell_{2}(\mathbb{N})\right)$ due to Enflo [12].

Surprisingly, AP is sufficient to answer both our question about the equality of the classes of approximable and compact operators and the injectivity of the map $J$ defined in Section 3.3.

Lemma 3.4.2 (Corollaries 4.8 and 4.13 in [33]). If $X^{*}$ or $Y$ has AP then $J$ is injective i.e.

$$
X^{*} \otimes_{\pi} Y=\mathcal{N}(X, Y)
$$

and

$$
\mathcal{A}\left(X^{*}, Y\right)=X^{*} \otimes_{\varepsilon} Y=\mathcal{K}(X, Y) .
$$

For the second property, first recall the classical Radon-Nikodym Theorem:

Theorem 3.4.3 (Radon-Nikodym). Let $(M, \mathscr{A}, \mu)$ be a $\sigma$-finite measure space and $\nu \ll \mu$ be another measure on $(M, \mathscr{A})$. Then $\exists 0 \leq f \in L_{1}(M, \mu)$ s.t. $\forall A \in \mathscr{A}$

$$
\nu(A)=\int_{A} f d \mu .
$$

A Banach space $X$ has the Radon-Nikodym property (RNP) if the analogue of Theorem 3.4.3 "holds in $X$ ", here we replace $\nu$ with a vector measure $\nu: \mathscr{A} \rightarrow X$ of bounded variation which is absolutely continuous w.r.t a finite measure $\mu$ on $(M, \mathscr{A})$ and the integral with the Bochner integral. For details, see Chapter 5 of [33]. The precise statement is not important to us, all we need are the following two results:

Proposition 3.4.4 (Corollary 5.4.2 in [33]). Every reflexive Banach space has RNP.

"Reasonable" spaces have AP, but spaces with RNP are more rare. Even $c_{0}(\mathbb{N})$ does not have RNP (Example 5.13 in [33]). 
The reason we introduced these properties is the following result:

Theorem 3.4.5 (5.33 in [33]). Let $X$ and $Y$ be Banach spaces s.t. $X^{*}$ has RNP and either $X^{*}$ or $Y^{*}$ has AP. Then

$$
\left(X \otimes_{\varepsilon} Y\right)^{*}=X^{*} \otimes_{\pi} Y^{*}
$$

Putting this together with Lemma 3.4.2 we have that, under the assumptions of Theorem 3.4.5,

$$
\mathcal{K}\left(X, Y^{*}\right)^{*}=\left(X \otimes_{\varepsilon} Y\right)^{*}=X^{*} \otimes_{\pi} Y^{*}=\mathcal{N}\left(X, Y^{*}\right)
$$

We are most interested in $L_{p}$ spaces, $p \in(1, \infty)$. They are reflexive and have AP, and hence

$$
\mathcal{K}\left(L_{p}\right)^{*}=\mathcal{K}\left(L_{p}, L_{q}^{*}\right)^{*}=\mathcal{N}\left(L_{p}, L_{q}^{*}\right)=\mathcal{N}\left(L_{p}\right)
$$

Combining this with the identification $\left(X \otimes_{\pi} Y\right)^{*}=B\left(X, Y^{*}\right)$, we obtain

$$
\mathcal{K}\left(L_{p}\right)^{* *}=\mathcal{N}\left(L_{p}\right)^{*}=\left(L_{p}^{*} \otimes_{\pi} L_{p}\right)^{*}=\left(L_{p} \otimes_{\pi} L_{p}^{*}\right)^{*}=B\left(L_{p}, L_{p}^{* *}\right)=B\left(L_{p}\right)
$$




\section{Chapter 4}

\section{Nuclear Operators}

We finally introduce the main object of study of this thesis: the algebra $\mathcal{N}\left(L_{p}(G)\right.$ ) of nuclear operators on $L_{p}(G)$ with a convolution-type product rather than the usual composition. This algebra was defined by Neufang to give the first example of a Banach algebra which is one-sided strongly Arens irregular (left but not right).

We do not study this algebra from that point of view until Chapter 6 . Here we define the convolution product on $\mathcal{N}\left(L_{p}(G)\right)$ and then realize $B\left(L_{p}(G)\right)$ as a Banach $L_{1}(G)$ - and then $\mathcal{N}\left(L_{p}(G)\right.$ )-module and study the operators obtained from these module actions. This algebra of operators turns out to have a very nice intrinsic characterization and is crucial to the results of Chapter 6 . 


\subsection{The convolution algebra of nuclear operators}

Recall that

$\mathcal{N}\left(L_{p}(G)\right)=\left\{\rho=\sum_{n=1}^{\infty} \xi_{n} \otimes \eta_{n} \in B\left(L_{p}(G)\right) \mid \sum_{n=1}^{\infty}\left\|\xi_{n}\right\|_{q}\left\|\eta_{n}\right\|_{p}<\infty\right\} \cong L_{q}(G) \otimes_{\pi} L_{p}(G)$

where for $f, \eta \in L_{p}(G)$ and $\xi \in L_{q}(G)$

$$
(\xi \otimes \eta) f:=\langle\xi, f\rangle \eta
$$

$\mathcal{N}\left(L_{p}(G)\right)$ is a two-sided ideal in $B\left(L_{p}(G)\right)$ since $\forall S, T \in B\left(L_{p}(G)\right)$

$$
S(\xi \otimes \eta) T=\left(T^{*} \xi\right) \otimes(S \eta)
$$

$\mathcal{N}\left(L_{p}(G)\right)$ inherits a norm from $L_{q}(G) \otimes_{\pi} L_{p}(G)$ which is given by

$$
\|\rho\|_{\mathcal{N}}:=\inf \left\{\sum_{n=1}^{\infty}\left\|\xi_{n}\right\|_{q}\left\|\eta_{n}\right\|_{p} \mid \rho=\sum_{n=1}^{\infty} \xi_{n} \otimes \eta_{n} \in \mathcal{N}\left(L_{p}(G)\right)\right\}
$$

For any $\rho \in \mathcal{N}\left(L_{p}(G)\right)$

$$
\|\rho\| \leq\|\rho\|_{\mathcal{N}}
$$

and $\forall S, T \in B\left(L_{p}(G)\right)$

$$
\|S \rho T\|_{\mathcal{N}} \leq\|S\|\|\rho\|_{\mathcal{N}}\|T\|
$$

$\mathcal{N}\left(L_{p}(G)\right)=\left(\mathcal{N}\left(L_{p}(G)\right), \circ,\|\cdot\|_{\mathcal{N}}\right)$ is a Banach algebra and $\mathcal{N}\left(L_{p}(G)\right)^{*}=B\left(L_{p}(G)\right)$ via the duality

$$
\langle T, \rho\rangle=\operatorname{tr} T \rho=\operatorname{tr} \rho T=\sum_{n=1}^{\infty}\left\langle\xi_{n}, T \eta_{n}\right\rangle
$$

for $T \in B\left(L_{p}(G)\right)$ and $\rho=\sum_{n=1}^{\infty} \xi_{n} \otimes \eta_{n}$ (this is indeed well-defined). 
We define a product on $\mathcal{N}\left(L_{p}(G)\right)$ via duality with $B\left(L_{p}(G)\right)$ which is different than composition and can be considered a convolution.

For $\rho, \tau \in \mathcal{N}\left(L_{p}(G)\right)$, and $T \in B\left(L_{p}(G)\right)$ define $T \odot \rho \in B\left(L_{p}(G)\right)$ and then $\rho * \tau \in \mathcal{N}\left(L_{p}(G)\right)$ by putting

$$
\begin{gathered}
T \odot \rho:=M_{\left\langle L_{t T L_{t}-1}, \rho\right\rangle} \in M_{\mathrm{RUC}(G)} \\
\langle T, \rho * \tau\rangle:=\langle T \odot \rho, \tau\rangle .
\end{gathered}
$$

Recall that for $f \in L_{\infty}(G), M_{f} \in B\left(L_{p}(G)\right)$ is the multiplication operator.

Proposition 4.1.1. $\mathcal{N}\left(L_{p}(G)\right)=\left(\mathcal{N}\left(L_{p}(G)\right), *,\|\cdot\|_{\mathcal{N}}\right)$ is a Banach algebra and $\odot$ makes $B\left(L_{p}(G)\right)$ a right Banach $\mathcal{N}\left(L_{p}(G)\right)$-module.

This result is due to Neufang (Definition und Satz 5.2.1 in [30]), and the gory details are checked in Proposition 3.1.2 in [3].

Embed $L_{\infty}(G)$ in $B\left(L_{p}(G)\right)$ as twisted multiplication operators via $\iota$ :

$$
L_{\infty}(G) \ni f \stackrel{\iota}{\mapsto} M_{\tilde{f}} \in B\left(L_{p}(G)\right) .
$$

$\iota$ is $w^{*}-w^{*}$-continuous and hence has preadjoint

$$
\pi: \mathcal{N}\left(L_{p}(G)\right)=B\left(L_{p}(G)\right)_{*} \rightarrow L_{\infty}(G)_{*}=L_{1}(G)
$$

$\pi$ is determined by

$$
\pi(\xi \otimes \eta)=\widetilde{\xi \eta}
$$

where $\xi \in L_{q}(G)$ and $\eta \in L_{p}(G)$.

We call $\pi$ the canonical quotient $\mathcal{N}\left(L_{p}(G)\right) \rightarrow L_{1}(G)$ due to the following result. 
Proposition 4.1.2 (Satz 5.3.1 in [30]). The map

$$
\pi:\left(\mathcal{N}\left(L_{p}(G)\right), *\right) \rightarrow\left(L_{1}(G), *\right)
$$

is an algebra homomorphism.

This is why we called $*$ a convolution product.

\section{2 $B\left(L_{p}(G)\right)$ as a Banach module}

We will make $B\left(L_{p}(G)\right)$ a left Banach $L_{1}(G)$ - and then, using Proposition 4.1.2, $\mathcal{N}\left(L_{p}(G)\right)$-module.

Proposition 4.2.1. $B\left(L_{p}(G)\right)$ is left Banach $L_{1}(G)$-module with module operation given by, for $f \in L_{1}(G)$ and $T \in B\left(L_{p}(G)\right)$, the weak integral

$$
f \star T:=\int_{G}^{w^{*}} L_{t^{-1}} T L_{t} f(t) d t
$$

Proof.

$$
\begin{aligned}
\|f \star T\| & =\sup _{\rho \in \mathcal{N}\left(L_{p}(G)\right)_{1}}|\langle f \star T, \rho\rangle| \\
& =\sup _{\rho \in \mathcal{N}\left(L_{p}(G)\right)_{1}}\left|\left\langle\int_{G}^{w^{*}} L_{t^{-1}} T L_{t} f(t) d t, \rho\right\rangle\right| \\
& =\sup _{\rho \in \mathcal{N}\left(L_{p}(G)\right)_{1}}\left|\int_{G}\left\langle L_{t^{-1}} T L_{t}, \rho\right\rangle f(t) d t\right| \\
& \leq \sup _{\rho \in \mathcal{N}\left(L_{p}(G)\right)_{1}} \int_{G}\left|\left\langle L_{t^{-1}} T L_{t}, \rho\right\rangle\right||f(t)| d t \\
& \leq \int_{G}\left\|L_{t^{-1}}\right\|\|T\|\left\|L_{t}\right\||f(t)| d t \\
& =\|T\| \int_{G}|f(t)| d t \\
& =\|f\|_{1}\|T\| .
\end{aligned}
$$


So the module map is submultiplicative for the norm.

The operation is bilinear and for any $f, h \in L_{1}(G), T \in B\left(L_{p}(G)\right), \rho \in \mathcal{N}\left(L_{p}(G)\right)$

$$
\begin{aligned}
& \langle h \star(f \star T), \rho\rangle=\left\langle h \star \int_{G}^{w^{*}} L_{t^{-1}} T L_{t} f(t) d t, \rho\right\rangle \\
& =\left\langle\int_{G}^{w^{*}} L_{s^{-1}}\left(\int_{G}^{w^{*}} L_{t^{-1}} T L_{t} f(t) d t\right) L_{s} h(s) d s, \rho\right\rangle \\
& =\int_{G}\left\langle L_{s^{-1}}\left(\int_{G}^{w^{*}} L_{t^{-1}} T L_{t} f(t) d t\right) L_{s}, \rho\right\rangle h(s) d s \\
& =\int_{G}\left\langle\left(\int_{G}^{w^{*}} L_{t^{-1}} T L_{t} f(t) d t\right), L_{s} \rho L_{s^{-1}}\right\rangle h(s) d s \\
& =\int_{G} \int_{G}\left\langle L_{t^{-1}} T L_{t}, L_{s} \rho L_{s^{-1}}\right\rangle f(t) d t h(s) d s \\
& =\int_{G} \int_{G}\left\langle L_{(s t)^{-1}} T L_{s t}, \rho\right\rangle f(t) d t h(s) d s \\
& =\int_{G} \int_{G}\left\langle L_{\left(s\left(s^{-1} t\right)\right)^{-1}} T L_{s\left(s^{-1} t\right)}, \rho\right\rangle f\left(s^{-1} t\right) d\left(s^{-1} t\right) h(s) d s \\
& =\int_{G} \int_{G}\left\langle L_{t^{-1}} T L_{t}, \rho\right\rangle f\left(s^{-1} t\right) d t h(s) d s \\
& =\int_{G} \int_{G}\left\langle L_{t^{-1}} T L_{t}, \rho\right\rangle h(s) f\left(s^{-1} t\right) d t d s \\
& =\int_{G} \int_{G}\left\langle L_{t^{-1}} T L_{t}, \rho\right\rangle h(s) f\left(s^{-1} t\right) d s d t \\
& =\int_{G}\left\langle L_{t^{-1}} T L_{t}, \rho\right\rangle \int_{G} h(s) f\left(s^{-1} t\right) d s d t \\
& =\int_{G}\left\langle L_{t^{-1}} T L_{t}, \rho\right\rangle(h * f)(t) d t \\
& =\left\langle\int_{G}^{w^{*}} L_{t^{-1}} T L_{t}(h * f)(t) d t, \rho\right\rangle \\
& =\langle(h * f) \star T, \rho\rangle .
\end{aligned}
$$

Proposition 4.2.2 (Satz 5.4.14 in $[30]$ ). $B\left(L_{p}(G)\right.$ ) is also a left Banach $\mathcal{N}\left(L_{p}(G)\right.$ )module via the module operation - defined by, for $\rho \in \mathcal{N}\left(L_{p}(G)\right)$ and $T \in B\left(L_{p}(G)\right)$,

$$
\rho \cdot T:=\pi(\rho) \star T=\int_{G}^{w^{*}} L_{t^{-1}} T L_{t} \pi(\rho)(t) d t
$$


and furthermore $\forall \rho, \tau \in \mathcal{N}\left(L_{p}(G)\right)$ and $T \in B\left(L_{p}(G)\right)$

$$
\langle\rho \cdot T, \tau\rangle=\langle T, \tau * \rho\rangle=\langle T \odot \tau, \rho\rangle
$$

If $G$ is discrete then we can take $\zeta \in \mathcal{N}\left(\ell_{p}(G)\right)$ s.t. $\pi(\zeta)=\delta_{e} \in \ell_{1}(G)$. Then $\forall T \in B\left(L_{p}(G)\right)$

$$
\zeta \cdot T=\pi(\zeta) \star T=\delta_{e} \star T=\int_{G} L_{t^{-1}} T L_{t} d \delta_{e}(t)=L_{e^{-1}} T L_{e}=T
$$

The following result will be used much later, but is interesting in its own right.

Lemma 4.2.3. The module operation $\cdot$ fixes $\mathcal{K}\left(L_{p}(G)\right)$, that is, $\rho \cdot K \in \mathcal{K}\left(L_{p}(G)\right)$ $\forall \rho \in \mathcal{N}\left(L_{p}(G)\right)$ and $K \in \mathcal{K}\left(L_{p}(G)\right)$.

Proof. Let $\rho \in \mathcal{N}\left(L_{p}(G)\right)$ and $K \in \mathcal{K}\left(L_{p}(G)\right)$. Since $\mathcal{K}\left(L_{p}(G)\right)^{* *}=B\left(L_{p}(G)\right)$, it suffices to show that $\rho \cdot K$ is $w^{*}$-continuous. Since $L_{p}(G)$ is reflexive and enjoys the Approximation Property, $\mathcal{K}\left(L_{p}(G)\right)=L_{q}(G) \otimes_{\varepsilon} L_{p}(G)$ has Mazur's property by [22] Corollary 5.2. So it suffices to consider a sequence $\tau_{n}$ in $\mathcal{N}\left(L_{p}(G)\right)_{1}$ with $\tau_{n} \underset{n}{\stackrel{w^{*}}{\rightarrow}} 0$.

$$
\begin{aligned}
\left\langle\rho \cdot K, \tau_{n}\right\rangle & =\left\langle\int_{G} L_{t^{-1}} K L_{t} \pi(\rho)(t) d t, \tau_{n}\right\rangle \\
& =\int_{G}\left\langle L_{t^{-1}} K L_{t}, \tau_{n}\right\rangle \pi(\rho)(t) d t \\
& =\int_{G} f_{n}(t) \pi(\rho)(t) d t
\end{aligned}
$$

with

$$
f_{n}(t):=\left\langle L_{t^{-1}} K L_{t}, \tau_{n}\right\rangle=\left\langle\tau_{n}, L_{t^{-1}} K L_{t}\right\rangle \rightarrow 0 \text { pointwise. }
$$


Then since

$$
\left|f_{n}(t)\right|=\left|\left\langle L_{t^{-1}} K L_{t}, \tau_{n}\right\rangle\right| \leq\left\|L_{t}^{-1}\right\|\|K\|\left\|L_{t}\right\|\left\|\tau_{n}\right\| \leq\|K\|
$$

and

$$
\int_{G}\|K\| \pi(\rho)(t) d t=\|K\|\|\pi(\rho)\|_{1}<\infty
$$

we have

$$
\left\langle\rho \cdot K, \tau_{n}\right\rangle=\int_{G} f_{n}(t) \pi(\rho)(t) d t \rightarrow 0
$$

by Lebesgue's Dominated Convergence Theorem.

\section{$4.3 \quad G$-continuous operators}

Definition 4.3.1. For a locally compact group $G$, let

$$
X\left(L_{p}(G)\right)=\left\{T \in B\left(L_{p}(G)\right) \mid G \ni t \mapsto L_{t^{-1}} T L_{t} \text { is }\|\cdot\| \text {-continuous }\right\}
$$

The elements of $X\left(L_{p}(G)\right)$ are called $G$-continuous operators.

Bekka formulated this definition for arbitrary representations of $G$ on $L_{2}(G)$ in [5].

$X\left(L_{p}(G)\right)$ is a closed unital subalgebra of $B\left(L_{p}(G)\right)$ which contains the compact (and hence nuclear, approximable, and finite rank) operators.

Proposition 4.3.2. $\mathcal{K}\left(L_{p}(G)\right) \subseteq X\left(L_{p}(G)\right)$.

Proof. Let $\xi \otimes \eta \in L_{q}(G) \otimes_{\varepsilon} L_{p}(G)=\mathcal{K}\left(L_{p}(G)\right)$ be a rank-one operator. 


$$
\begin{aligned}
\left\|L_{t^{-1}}(\xi \otimes \eta) L_{t}-\xi \otimes \eta\right\| & =\left\|\left(L_{t}^{*} \xi\right) \otimes\left(L_{t^{-1}} \eta\right)-\xi \otimes \eta\right\| \\
& =\left\|\left(L_{t^{-1}} \xi\right) \otimes\left(L_{t^{-1}} \eta\right)-\xi \otimes \eta\right\| \\
& \leq\left\|\left(L_{t^{-1}} \xi\right) \otimes\left(L_{t^{-1}} \eta\right)-\left(L_{t^{-1}} \xi\right) \otimes \eta\right\|+\left\|\left(L_{t^{-1}} \xi\right) \otimes \eta-\xi \otimes \eta\right\| \\
& =\left\|\left(L_{t^{-1}} \xi\right) \otimes\left(L_{t^{-1}} \eta-\eta\right)\right\|+\left\|\left(L_{t^{-1}} \xi-\xi\right) \otimes \eta\right\| \\
& =\left\|L_{t^{-1}}\right\|\|\xi\|_{q}\left\|L_{t^{-1}} \eta-\eta\right\|_{p}+\left\|L_{t^{-1}} \xi-\xi\right\|_{q}\|\eta\|_{p} \\
& \rightarrow 0 \quad \text { as } \quad t \rightarrow e
\end{aligned}
$$

by the strong continuity of left translations [14, Proposition 2.41]. So $X\left(L_{p}(G)\right)$ contains $\mathcal{F}\left(L_{p}(G)\right)$ and hence also

$$
\overline{\mathcal{F}\left(L_{p}(G)\right)}=\mathcal{A}\left(L_{p}(G)\right)=\mathcal{K}\left(L_{p}(G)\right) .
$$

The $G$-continuous operators are exactly those arising from the module action of $L_{1}(G)$ on $B\left(L_{p}(G)\right)$ defined in Proposition 4.2.1.

Lemma 4.3.3. $X\left(L_{p}(G)\right)=L_{1}(G) \star B\left(L_{p}(G)\right)$.

Proof. We extend Bekka's proof of this result for $p=2[4$, Lemma 5.2]. First, let $f \in L_{1}(G), T \in B\left(L_{p}(G)\right)$, and $s, t \in G$. We calculate

$$
\begin{aligned}
L_{t^{-1}}(f \star T) L_{t} & =L_{t^{-1}}\left(\int_{G}^{w^{*}} L_{s^{-1}} T L_{s} f(s) d t\right) L_{t} \\
& =\int_{G}^{w^{*}} L_{(t s)^{-1}} T L_{t s} f(s) d t \\
& =\int_{G}^{w^{*}} L_{s^{-1}} T L_{s} f\left(t^{-1} s\right) d\left(t^{-1} s\right) \\
& =\int_{G}^{w^{*}} L_{s^{-1}} T L_{s}\left(L_{t^{-1}} f\right)(s) d s \\
& =\left(L_{t^{-1}} f\right) \star T .
\end{aligned}
$$


Then

$$
\left\|L_{t^{-1}}(f \star T) L_{t}-f \star T\right\|=\left\|\left(L_{t^{-1}} f\right) \star T-f \star T\right\| \leq\left\|L_{t^{-1}} f-f\right\|_{1}\|T\| \rightarrow 0
$$

as $t \rightarrow e$ by the strong continuity of left translations. Thus $f \star T \in X\left(L_{p}(G)\right)$ and hence

$$
L_{1}(G) \star B\left(L_{p}(G)\right) \subseteq X\left(L_{p}(G)\right)
$$

Now take $T \in X\left(L_{p}(G)\right)$. Let $\left(e_{\alpha}\right)$ be the standard BAI of $L_{1}(G)$ (see Proposition 2.42 in [14]). Then

$$
\begin{aligned}
\left\|e_{\alpha} \star T-T\right\| & =\left\|\int_{G}^{w^{*}} L_{t^{-1}} T L_{t} e_{\alpha}(t) d t-T \int_{G} e_{\alpha}(t) d t\right\| \\
& =\left\|\int_{G}^{w^{*}} L_{t^{-1}} T L_{t} e_{\alpha}(t) d t-\int_{G}^{w^{*}} T e_{\alpha}(t) d t\right\| \\
& =\left\|\int_{G}^{w^{*}}\left(L_{t^{-1}} T L_{t}-T\right) e_{\alpha}(t) d t\right\| \\
& =\left\|\int_{\text {supp } e_{\alpha}}^{w^{*}}\left(L_{t^{-1}} T L_{t}-T\right) e_{\alpha}(t) d t\right\| \\
& \leq \int_{\text {supp } e_{\alpha}}\left\|L_{t^{-1}} T L_{t}-T\right\| e_{\alpha}(t) \mid d t \\
& \rightarrow 0
\end{aligned}
$$

since $T \in X\left(L_{p}(G)\right)$. So $T \in \overline{L_{1}(G) \star B\left(L_{p}(G)\right)}$ and by Cohen's Factorization Theorem,

$$
X\left(L_{p}(G)\right) \subseteq \overline{L_{1}(G) \star B\left(L_{p}(G)\right)}=L_{1}(G) \star B\left(L_{p}(G)\right) .
$$

Theorem 4.3.4. $X\left(L_{p}(G)\right)=\mathcal{N}\left(L_{p}(G)\right) \cdot B\left(L_{p}(G)\right)$. 
Proof. Indeed,

$$
\begin{aligned}
X\left(L_{p}(G)\right) & =L_{1}(G) \star B\left(L_{p}(G)\right) \\
& =\left\{f \star T \mid f \in L_{1}(G), T \in B\left(L_{p}(G)\right)\right\} \\
& =\left\{\pi(\rho) \star T \mid \rho \in \mathcal{N}\left(L_{p}(G)\right), T \in B\left(L_{p}(G)\right)\right\} \\
& =\left\{\rho \cdot T \mid \rho \in \mathcal{N}\left(L_{p}(G)\right), T \in B\left(L_{p}(G)\right)\right\} \\
& =\mathcal{N}\left(L_{p}(G)\right) \cdot B\left(L_{p}(G)\right) .
\end{aligned}
$$

We now define an isometric antirepresentation of $X\left(L_{p}(G)\right)^{*}$. First we need a product on $X\left(L_{p}(G)\right)^{*}$. Let $\iota: X\left(L_{p}(G)\right) \hookrightarrow B\left(L_{p}(G)\right)$ be the embedding and $\iota^{*}: B\left(L_{p}(G)\right)^{*} \rightarrow X\left(L_{p}(G)\right)^{*}$ be the quotient. We can define a product on $X\left(L_{p}(G)\right)$ via the second Arens product on $B\left(L_{p}(G)\right)^{*}=\mathcal{N}\left(L_{p}(G)\right)^{* *}$ and $\iota^{*-1}$.

This product is given explicitly by

$$
\begin{aligned}
& \langle m n, T\rangle=\langle n, T m\rangle \\
& \langle T m, \rho\rangle=\langle m, \rho \cdot T\rangle
\end{aligned}
$$

for $m, n \in X\left(L_{p}(G)\right)^{*}, T \in X\left(L_{p}(G)\right), \rho \in \mathcal{N}\left(L_{p}(G)\right)$.

Theorem 4.3.5. The map

$$
\Gamma: X\left(L_{p}(G)\right)^{*} \hookrightarrow B_{\mathcal{N}\left(L_{p}(G)\right)}\left(B\left(L_{p}(G)\right)\right)
$$

given by for $m \in X\left(L_{p}(G)\right)^{*}, T \in B\left(L_{p}(G)\right)$ and $\rho \in \mathcal{N}\left(L_{p}(G)\right)$

$$
\langle\Gamma(m) T, \rho\rangle=\langle m, \rho \cdot T\rangle
$$

is an isometric, $w^{*}-w^{*}$-continuous antihomomorphism. 
Proof. We extend Neufang's proof of this result for $p=2$ from [27], breaking the proof up into 4 steps.

(i) First, we show that $\Gamma\left(X\left(L_{p}(G)\right)^{*}\right) \subseteq B_{\mathcal{N}\left(L_{p}(G)\right)}\left(B\left(L_{p}(G)\right)\right)$. Clearly

$$
\Gamma\left(X\left(L_{p}(G)\right)^{*}\right) \subseteq B\left(B\left(L_{p}(G)\right)\right)
$$

Let $m \in X\left(L_{p}(G)\right)^{*}, \rho, \tau \in \mathcal{N}\left(L_{p}(G)\right)$, and $T \in B\left(L_{p}(G)\right)$. Then

$$
\begin{aligned}
\langle\Gamma(m)(\tau \cdot T), \rho\rangle & =\langle m, \rho \cdot(\tau \cdot T)\rangle \\
& =\langle m,(\rho * \tau) \cdot T\rangle \\
& =\langle\Gamma(m) T, \rho * \tau\rangle \\
& =\langle\tau \cdot \Gamma(m) T, \rho\rangle .
\end{aligned}
$$

Hence

$$
\Gamma(m)(\tau \cdot T)=\tau \cdot \Gamma(m) T
$$

(ii) To see that $\Gamma$ is $w^{*}$-w*-continuous, let $X\left(L_{p}(G)\right)^{*} \ni m_{\alpha} \underset{\alpha}{\stackrel{w^{*}}{\longrightarrow}} 0$ and

$T \otimes \rho \in B\left(L_{p}(G)\right) \otimes_{\pi} \mathcal{N}\left(L_{p}(G)\right) \stackrel{!}{=} B\left(B\left(L_{p}(G)\right), \mathcal{N}\left(L_{p}(G)\right)^{*}\right)_{*}=B\left(B\left(L_{p}(G)\right)\right)_{*}$

with the equality marked "!" by Proposition 3.3.2. Then

$$
\left\langle\Gamma\left(m_{\alpha}\right), T \otimes \rho\right\rangle=\left\langle\Gamma\left(m_{\alpha}\right) T, \rho\right\rangle=\left\langle m_{\alpha}, \rho \cdot T\right\rangle \rightarrow 0
$$

Hence

$$
\Gamma\left(m_{\alpha}\right) \underset{\alpha}{\stackrel{w^{*}}{\longrightarrow}} 0
$$


(iii) $\Gamma$ is isometric, since

$$
\begin{aligned}
\|\Gamma(m)\| & =\sup _{T \in B\left(L_{p}(G)\right)_{1}}\|\Gamma(m) T\| \\
& =\sup _{T \in B\left(L_{p}(G)\right)_{1}} \sup _{\rho \in \mathcal{N}\left(L_{p}(G)\right)_{1}}|\langle\Gamma(m) T, \rho\rangle| \\
& =\sup _{T \in B\left(L_{p}(G)\right)_{1}} \sup _{\rho \in \mathcal{N}\left(L_{p}(G)\right)_{1}}|\langle m, \rho \cdot T\rangle| \\
& \leq \sup _{T \in B\left(L_{p}(G)\right)_{1}} \sup _{\rho \in \mathcal{N}\left(L_{p}(G)\right)_{1}}\|m\|\|\rho\|\|T\| \\
& =\|m\|
\end{aligned}
$$

and for the opposite inequality, take $\left(\rho_{\alpha}\right) \subset \mathcal{N}\left(L_{p}(G)\right)_{1}$ s.t. $\left(\pi\left(\rho_{\alpha}\right)\right)=\left(e_{\alpha}\right)$ the standard approximate identity of $L_{1}(G)$. Then $\forall T \in B\left(L_{p}(G)\right)$

$$
\rho_{\alpha} \cdot T=\pi\left(\rho_{\alpha}\right) \star T=e_{\alpha} \star T \rightarrow T
$$

So

$$
\begin{aligned}
\|m\| & =\sup _{T \in X\left(L_{p}(G)\right)_{1}}|\langle m, T\rangle| \\
& =\sup _{T \in X\left(L_{p}(G)\right)_{1}}\left|\left\langle m, \lim _{\alpha} \rho_{\alpha} \cdot T\right\rangle\right| \\
& =\sup _{T \in X\left(L_{p}(G)\right)_{1}} \lim _{\alpha}\left|\left\langle m, \rho_{\alpha} \cdot T\right\rangle\right| \\
& =\sup _{T \in X\left(L_{p}(G)\right)_{1}} \lim _{\alpha}\left|\left\langle\Gamma(m) T, \rho_{\alpha}\right\rangle\right| \\
& \leq \sup _{T \in X\left(L_{p}(G)\right)_{1}} \lim _{\alpha}\|\Gamma(m) T\|\left\|\rho_{\alpha}\right\| \\
& =\sup _{T \in X\left(L_{p}(G)\right)_{1}}\|\Gamma(m) T\| \\
& \leq \sup _{T \in X\left(L_{p}(G)\right)_{1}}\|\Gamma(m)\|\|T\| \\
& =\|\Gamma(m)\| .
\end{aligned}
$$


(iv) Finally, to show that $\Gamma$ is an antihomomorphism, note that $\forall m \in X\left(L_{p}(G)\right)^{*}$, $T \in X\left(L_{p}(G)\right), \rho, \tau \in \mathcal{N}\left(L_{p}(G)\right)$,

$$
\langle(\rho \cdot T) m, \tau\rangle=\langle m,(\tau * \rho) \cdot T\rangle=\langle\Gamma(m) T, \tau * \rho\rangle=\langle\rho \cdot(\Gamma(m) T), \tau\rangle
$$

hence

$$
(\rho \cdot T) m=\rho \cdot(\Gamma(m) T)
$$

and then

$$
\langle\Gamma(m n) T, \rho\rangle=\langle m n, \rho \cdot T\rangle=\langle n,(\rho \cdot T) m\rangle=\langle n, \rho(\Gamma(m) T)\rangle=\langle\Gamma(n) \Gamma(m) T, \rho\rangle
$$

which gives

$$
\Gamma(m n)=\Gamma(n) \Gamma(m)
$$




\section{Chapter 5}

\section{Spiral Nebulae}

This chapter is entirely dedicated to an obscure technical construction first defined on von Neumann algebras by Hofmeier and Wittstock in [21] and an automatically normality result for which it is built. Neufang exploited this construction to yield a 2-point DTC set for $\operatorname{LUC}(G)^{*}$ in [29]. We have to work pretty hard to adapt the definition and result into our context, and we use another obscure and shockingly similar construction for locally compact groups first defined by Grosser and Losert in [16] - it seems almost designed for this purpose.

Throughout this chapter, the symbol " \pm " is meant to be replaced with either + or -, consistently. For example, the statement

$$
f\left(x_{ \pm}\right)=y_{ \pm}
$$

is to be read as

$$
y_{+}=f\left(x_{+}\right) \quad \text { and } \quad y_{-}=f\left(x_{-}\right) \text {. }
$$




\subsection{Spiral nebulae on von Neumann algebras}

In order to formulate the definition we will extend, we must first introduce some terminology from von Neumann algebra theory.

A von Neumann algebra is a WOT-closed unital *-subalgebra of $B(\mathcal{H})$ for some Hilbert space $\mathcal{H}$. A von Neumann algebra has a (unique) predual and hence a $w^{*}$ topology. An idempotent $e \in \mathcal{M}$ a von Neumann algebra is a projection if $e^{*}=e$. A projection $p \neq 0$ is minimal if $q \leq p \Rightarrow q=0$ or $p$ and of finite rank if it is the sum of finitely many minimal projections. A $*$-automorphism $\varphi$ of $\mathcal{M}$ is an automorphism of $\mathcal{M}$ s.t. $\varphi\left(x^{*}\right)=\varphi(x)^{*} \quad \forall x \in \mathcal{M}$. Note that $*$-automorphisms preserve projections.

Definition 5.1.1 (1.1 of [21]). Let $\mathcal{M}$ be a von Neumann algebra. An atomic countable spiral nebula on $\mathcal{M}$ is a sequence $\left(\alpha_{n}, e_{n}\right)_{n \in \mathbb{Z}}$ of $*$-automorphisms $\alpha_{n}$ of $\mathcal{M}$ and finite rank projections $e_{n} \in \mathcal{M}$ which satisfy:

(i) $e_{0} \leq e_{n} \leq e_{n+1}$ and $e_{0} \leq e_{-n} \leq e_{-(n+1)} \quad \forall n \in \mathbb{N}$.

(ii) $w^{*}-\lim _{n \rightarrow \infty} e_{n}=w^{*}-\lim _{n \rightarrow \infty} e_{-n}=\mathrm{id}_{\mathcal{M}}$.

(iii) $\alpha_{m}\left(e_{m}\right) \perp \alpha_{n}\left(e_{n}\right)$ and $\alpha_{-m}\left(e_{-m}\right) \perp \alpha_{-n}\left(e_{-n}\right) \quad \forall m, n \in \mathbb{N}, m \neq n$.

(iv) $\alpha_{m}^{-1} \alpha_{n}\left(e_{n}\right) \perp \alpha_{-k}^{-1} \alpha_{-l}\left(e_{-l}\right) \quad \forall m, n, k, l \in \mathbb{N}, m<n$ and $k<l$.

The utility of this bizarre construction is the following automatic normality result.

Theorem 5.1.2 (Lemma 1.4(ii) in [21]). Let $\left(\alpha_{n}, e_{n}\right)_{n \in \mathbb{Z}}$ be an atomic countable spiral nebula on $\mathcal{M}$ and let $\Psi_{ \pm}$be arbitrary $w^{*}$-cluster points of $\left(\alpha_{ \pm n}^{-1}\right)_{n \in \mathbb{N}_{0}}$. Let $\Phi \in B(\mathcal{M})$ s.t. $\alpha_{n} \Phi=\Phi \alpha_{n} \forall n \in \mathbb{Z}$. Then $\Phi$ is normal iff

$$
\Psi_{ \pm} \Phi=\Phi \Psi_{ \pm}
$$


The meat of the proof of Theorem 5.1.2 is the following:

Lemma 5.1.3. Let $\left(\alpha_{n}, e_{n}\right)_{n \in \mathbb{Z}}$ be an atomic countable spiral nebula on a von Neumann algebra $\mathcal{M}$ and let $\Psi_{ \pm}$be arbitrary $w^{*}$-cluster points of $\left(\alpha_{ \pm n}^{-1}\right)_{n \in \mathbb{N}_{0}}$. Let $\Phi \in B(\mathcal{M})$ s.t. $\alpha_{n} \Phi=\Phi \alpha_{n} \forall n \in \mathbb{Z}$. Then if

$$
\Psi_{ \pm} \Phi=\Phi \Psi_{ \pm}
$$

and $\forall n \in \mathbb{Z}$

$$
\Phi\left(e_{n} \mathcal{M} e_{n}\right)=0
$$

then $\Phi=0$.

\subsection{Spiral nebulae on $B\left(L_{p}(G)\right)$}

We would like to generalize the notion of a countable spiral nebula on a von Neumann algebra to a spiral nebula of arbitrary cardinality on a Banach algebra $\mathfrak{A}$. Fortunately, the involutive structure of the von Neumann algebra and countability of the nebula are not essential. However, it is apparent that $\mathfrak{A}$ must at least be a dual Banach algebra (in the sense of Runde [32], that is, a Banach algebra which is a dual space and such that the multiplication is separately $w^{*}$-w*-continuous). Roughly, dual Banach algebras are to Banach algebras as von Neumann algebras are to $\mathrm{C}^{*}$-algebras. One could indeed formulate a definition for dual Banach algebras, but von Neumann algebras and $\mathrm{C}^{*}$-algebras enjoy many properties even dual Banach algebras do not (necessarily), many of which are implicit in the proof of Theorem 5.1.2. For example, any $*$-automorphism of a $\mathrm{C}^{*}$-algebra is automatically a contraction, but this is not true for dual Banach algebras. Thus either much stronger or more concrete assumptions are needed. 
Since we are mainly interested in $\mathcal{N}\left(L_{p}(G)\right.$ ), we will (with an eye towards commuting representations on $\left.B\left(L_{p}(G)\right)\right)$ only define spiral nebulae on $B\left(L_{p}(G)\right)$ and we will require that the automorphisms and idempotents (rather than projections) are very concrete. Property (iv) in Definition 5.1.1 needs to be strengthened and a strengthened property (i) is implicitly used to direct our index set.

Definition 5.2.1. A compact spiral nebula on $\mathfrak{A}:=B\left(L_{p}(G)\right)$ is a collection $\left(\alpha_{ \pm i}, e_{ \pm i}\right)_{i \in I}$ of automorphisms $\alpha_{ \pm i}=\operatorname{Ad}\left(L_{t_{ \pm i}}\right)$ of $\mathfrak{A}$ for some $t_{ \pm i} \in G$ and multiplication operators $e_{ \pm i}$ of characteristic functions of compact subsets of $G$ which satisfy:

(i) $w^{*}-\lim _{i} e_{ \pm i}=\mathrm{id}_{\mathfrak{A}}$ where $I$ is directed by inclusion of the compact sets.

(ii) $\alpha_{ \pm i}\left(e_{ \pm i}\right) \perp \alpha_{ \pm j}\left(e_{ \pm j}\right) \quad \forall i \neq j \in I$.

(iii) $\alpha_{+j}^{-1} \alpha_{+i}\left(e_{+i}\right) \perp \alpha_{-l}^{-1} \alpha_{-k}\left(e_{-k}\right) \quad \forall i, j, k, l \in I, i \neq j$ and $k \neq l$.

Thankfully, compact spiral nebulae do exist.

Theorem 5.2.2. Let $p \in(1, \infty)$ and $G$ be a locally compact non-compact group. Then $B\left(L_{p}(G)\right)$ admits a compact spiral nebula.

Proof. By Lemma 3 in [25] (which is based on Lemma 1 in [24]), there exists a family of compact subsets $\left(K_{i}\right)_{i \in I}$ of $G$ and families of group elements $\left(y_{i}\right)_{i \in I}$ and $\left(z_{i}\right)_{i \in I}$ s.t.

(i) $\left\{K_{i}: i \in I\right\}$ is closed under finite unions.

(ii) $\bigcup_{i \in I} K_{i}^{\circ}=G$.

(iii) the families $\left\{K_{i} y_{i}: i \in I\right\}$ and $\left\{K_{i} z_{i}: i \in I\right\}$ are pairwise disjoint.

(iv) $K_{i} y_{i} y_{j}^{-1} \cap K_{k} z_{k} z_{l}^{-1}=\varnothing$ if $i \neq j, k \neq l, i, j, k, l \in I$. 
For $i \in I$ let

$$
e_{ \pm i}:=M \chi_{K_{i}^{-1}}, \quad \alpha_{+i}:=\operatorname{Ad}\left(L_{y_{i}}\right), \quad \text { and } \quad \alpha_{-i}:=\operatorname{Ad}\left(L_{z_{i}}\right)
$$

Then properties (ii) and (iii) in Definition 5.2.1 are satisfied. It remains to show property (i). Let $\Lambda$ be a the collection of all compact subsets of $G$, directed by inclusion. For $K \in \Lambda$ let $M_{K}$ be the multiplication operator $M \chi_{K} \in B\left(L_{p}(G)\right)$. By Proposition 7.3 .7 in [7],

$$
M_{K} \stackrel{S O T}{\longrightarrow} \mathrm{id}_{\mathfrak{A}} \Rightarrow M_{K} \stackrel{w^{*}}{\rightarrow} \mathrm{id}_{\mathfrak{A}}
$$

So it suffices to show that $\kappa:=\left\{K_{i}^{-1}\right\}_{i \in I}$ is a subnet of $\Lambda$. We need only verify that $\kappa$ is cofinal in $\Lambda$, so take $K \in \Lambda$. Then $\left\{\left(K_{i}^{-1}\right)^{\circ}: i \in I\right\}$ is an open cover of $K$, so since $K$ is compact $\exists$ a finite subcover $\left\{\left(K_{i_{1}}^{-1}\right)^{\circ}, \ldots,\left(K_{i_{n}}^{-1}\right)^{\circ}\right\}$. So

$$
K \subseteq \bigcup_{j=1}^{n}\left(K_{i_{j}}^{-1}\right)^{\circ} \subseteq \bigcup_{j=1}^{n}\left(K_{i_{j}}^{-1}\right) \in \kappa
$$

since $\kappa$ is closed under finite unions.

\subsection{The automatic normality result}

In this section there are many results and there is little flow between them, so they are named as follows: Lemmata are independent of each other and are used to prove the Theorem (5.3.8), and Propositions are used to prove the Lemmata.

Throughout this section $\left(\alpha_{ \pm i}, e_{ \pm i}\right)_{i \in I}$ is a compact spiral nebula on $\mathfrak{A}:=B\left(L_{p}(G)\right)$ as defined in Definition 5.2.1 and $\Psi_{ \pm}$are arbitrary $w^{*}$-cluster points of $\left(\alpha_{ \pm i}\right)_{i \in I}$. 
Proposition 5.3.1. Let $f \in L_{p}(G)$. Then

$$
\sum_{i \in I}\left\|\alpha_{ \pm i}\left(e_{ \pm i}\right) f\right\|_{p}^{p} \leq\|f\|_{p}^{p}
$$

Proof. Let $f \in L_{p}(G)$ and $F \subset I$ be finite. First note that $\forall i \in I$,

$$
\alpha_{ \pm i}\left(e_{ \pm i}\right)=M \chi_{y_{ \pm i} K_{ \pm i}}
$$

for some $y_{ \pm i} \in G$ and compact $K_{ \pm i} \subset G$ s.t.

$$
y_{ \pm i} K_{ \pm i} \cap y_{ \pm j} K_{ \pm j}=\varnothing \quad \text { if } \quad i \neq j
$$

Now

$$
\begin{aligned}
\sum_{i \in F}\left\|\alpha_{ \pm i}\left(e_{ \pm i}\right) f\right\|_{p}^{p} & =\sum_{i \in F}\left\|M \chi_{y_{ \pm i} K_{ \pm i}} f\right\|_{p}^{p} \\
& =\sum_{i \in F} \int_{G}\left|\chi_{y_{ \pm} K_{ \pm i}} f\right|^{p} \\
& =\sum_{i \in F} \int_{G} \chi_{y_{ \pm i} K_{ \pm i}}|f|^{p} \\
& =\sum_{i \in F} \int_{y_{ \pm i} K_{ \pm i}}|f|^{p} \\
& =\int_{\bigcup_{i \in F} y_{ \pm i} K_{ \pm i}}|f|^{p} \quad \text { since the } y_{ \pm i} K_{ \pm i} \text { are disjoint } \\
& \leq \int_{G}|f|^{p} \\
& =\|f\|_{p}^{p} .
\end{aligned}
$$

Then since this is true for all finite $F \subset I$ the result follows. 
Lemma 5.3.2. Let $C \subseteq I$. The operators $\Theta_{ \pm C}: \mathfrak{A} \rightarrow \mathfrak{A}$ defined by

$$
\Theta_{ \pm C}(T):=\sum_{i \in I \backslash C} \alpha_{ \pm i}\left(e_{ \pm i} x e_{ \pm i}\right)
$$

are contractions.

For convenience, we will write

$$
\Theta_{ \pm}:=\Theta_{ \pm \varnothing}
$$

and for $j \in I$,

$$
\Theta_{ \pm j}:=\Theta_{ \pm\{j\}} .
$$

Proof. It suffices to prove the result for $C=\varnothing$. Let $\xi \otimes \eta \in L_{q}(G) \otimes_{\pi} L_{p}(G)=\mathfrak{A}_{*}$ be an elementary tensor and $x \in \mathfrak{A}_{1}$. Then

$$
\begin{aligned}
\left|\left\langle\Theta_{ \pm}(x), \xi \otimes \eta\right\rangle\right| & =\left|\left\langle\Theta_{ \pm}(x) \xi, \eta\right\rangle\right| \\
& =\left|\int_{G}\left(\Theta_{ \pm}(x) \xi\right) \eta\right| \\
& \leq \int_{G}\left|\left(\Theta_{ \pm}(x) \xi\right) \eta\right| \\
& =\int_{G}\left|\sum_{i \in I}\left(\alpha_{ \pm i}\left(e_{ \pm i} x e_{ \pm i}\right) \xi\right) \eta\right| \\
& =\int_{G}\left|\sum_{i \in I}\left(\alpha_{ \pm i}\left(e_{ \pm i}\right) \alpha_{ \pm i}(x) \alpha_{ \pm i}\left(e_{ \pm i}\right) \xi\right) \eta\right| \\
& =\int_{G}\left|\sum_{i \in I} M \chi_{y_{ \pm i} K_{ \pm i}}\left(\alpha_{ \pm i}(x) \alpha_{ \pm i}\left(e_{ \pm i}\right) \xi\right) \eta\right| \\
& =\int_{G}\left|\sum_{i \in I}\left(\alpha_{ \pm i}(x) \alpha_{ \pm i}\left(e_{ \pm i}\right) \xi\right) M \chi_{y_{i} K_{ \pm i}} \eta\right| \\
& =\int_{G}\left|\sum_{i \in I}\left(\alpha_{ \pm i}(x) \alpha_{ \pm i}\left(e_{ \pm i}\right) \xi\right) \alpha_{ \pm i}\left(e_{ \pm i}\right) \eta\right|
\end{aligned}
$$




$$
\begin{aligned}
& =\left\|\sum_{i \in I}\left(\alpha_{ \pm i}(x) \alpha_{ \pm i}\left(e_{ \pm i}\right) \xi\right) \alpha_{ \pm i}\left(e_{ \pm i}\right) \eta\right\|_{1} \\
& \leq \sum_{i \in I}\left\|\left(\alpha_{ \pm i}(x) \alpha_{ \pm i}\left(e_{ \pm i}\right) \xi\right) \alpha_{ \pm i}\left(e_{ \pm i}\right) \eta\right\|_{1} \\
& \leq \sum_{i \in I}\left\|\alpha_{ \pm i}(x) \alpha_{ \pm i}\left(e_{ \pm i}\right) \xi\right\|_{p}\left\|\alpha_{ \pm i}\left(e_{ \pm i}\right) \eta\right\|_{q} \\
& \leq \sum_{i \in I}\left\|\alpha_{ \pm i}\right\|\|x\|\left\|\alpha_{ \pm i}\left(e_{ \pm i}\right) \xi\right\|_{p}\left\|\alpha_{ \pm i}\left(e_{ \pm i}\right) \eta\right\|_{q} \\
& \leq \sum_{i \in I}\left\|\alpha_{ \pm i}\left(e_{ \pm i}\right) \xi\right\|_{p}\left\|\alpha_{ \pm i}\left(e_{ \pm i}\right) \eta\right\|_{q} \\
& \leq\left(\sum_{i \in I}\left\|\alpha_{ \pm i}\left(e_{ \pm i}\right) \xi\right\|_{p}^{p}\right)^{1 / p}\left(\sum_{i \in I}\left\|\alpha_{ \pm i}\left(e_{ \pm i}\right) \eta\right\|_{q}^{q}\right)^{1 / q} \\
& \leq\|\xi\|_{q}\|\eta\|_{p} \quad \text { by Proposition 5.3.1. }
\end{aligned}
$$

Now take $\rho \in L_{q}(G) \otimes_{\pi} L_{p}(G)$ and $\varepsilon>0$ and let $\sum_{n=1}^{\infty} \xi_{n} \otimes \eta_{n}$ be a representation of $\rho$ with

$$
\sum_{n=1}^{\infty}\left\|\xi_{n}\right\|_{q}\left\|\eta_{n}\right\|_{p}<\|\rho\|_{\pi}+\varepsilon
$$

Then

$$
\begin{aligned}
\left|\left\langle\Theta_{ \pm}(x), \rho\right\rangle\right| & =\left|\left\langle\Theta_{ \pm}(x), \sum_{n=1}^{\infty} \xi_{n} \otimes \eta_{n}\right\rangle\right| \\
& \leq \sum_{n=1}^{\infty}\left|\left\langle\Theta_{ \pm}(x), \xi_{n} \otimes \eta_{n}\right\rangle\right| \\
& \leq \sum_{n=1}^{\infty}\left\|\xi_{n}\right\|\left\|_{q}\right\| \eta_{n} \|_{p} \\
& <\|\rho\|_{\pi}+\varepsilon
\end{aligned}
$$

and since $\varepsilon>0$ was arbitrary,

$$
\left|\left\langle\Theta_{ \pm}(x), \rho\right\rangle\right| \leq\|\rho\|_{\pi}
$$


Finally

$$
\left\|\Theta_{ \pm}\right\|=\sup _{x \in \mathfrak{A}_{1}}\left\|\Theta_{ \pm}(x)\right\|=\sup _{x \in \mathfrak{A}_{1}} \sup _{\rho \in \mathfrak{A}_{* 1}}\left|\left\langle\Theta_{ \pm}(x), \rho\right\rangle\right| \leq 1
$$

Lemma 5.3.3. $\Psi_{ \pm} \Theta_{ \pm}=\mathrm{id}_{\mathfrak{A}}$.

Proof. Let $x \in \mathfrak{A}$ and $i, j, k \in I, i \preceq j$ (i.e. $K_{i} \subseteq K_{j}$ ) and first calculate

$$
\begin{aligned}
e_{ \pm i}\left(\alpha_{ \pm j}^{-1} \alpha_{ \pm k}\left(e_{ \pm k} x e_{ \pm k}\right)\right) e_{ \pm i} & =e_{ \pm i} e_{ \pm j}\left(\alpha_{ \pm j}^{-1} \alpha_{ \pm k}\left(e_{ \pm k} x e_{ \pm k}\right)\right) e_{ \pm i} \\
& =e_{ \pm i} \alpha_{ \pm j}^{-1}\left(\underline{\alpha_{ \pm j}\left(e_{ \pm j}\right) \alpha_{ \pm k}\left(e_{ \pm k}\right)} \alpha_{ \pm k}\left(x e_{ \pm k}\right)\right) e_{ \pm i} \\
& =e_{ \pm i} \alpha_{ \pm j}^{-1}\left(\underline{\delta_{j, k}} \alpha_{ \pm k}\left(x e_{ \pm k}\right)\right) e_{ \pm i} \\
& =\delta_{j, k} e_{ \pm i}\left(\alpha_{ \pm j}^{-1} \alpha_{ \pm k}\left(e_{ \pm k} x e_{ \pm k}\right)\right) e_{ \pm i} \\
& =\delta_{j, k} e_{ \pm i} e_{ \pm j} x e_{ \pm j} e_{ \pm i} \\
& =\delta_{j, k} e_{ \pm i} x e_{ \pm i}
\end{aligned}
$$

using property (ii) of Definition 5.2.1. Now

$$
\begin{aligned}
e_{ \pm i}\left(\Psi_{ \pm} \Theta_{ \pm}(x)\right) e_{ \pm i} & =e_{ \pm i}\left(w^{*}-\lim _{j} \alpha_{ \pm j}^{-1}\left(w^{*}-\sum_{k \in I} \alpha_{ \pm k}\left(e_{ \pm k} x e_{ \pm k}\right)\right)\right) e_{ \pm i} \\
& =w^{*}-\lim _{j} w^{*}-\sum_{k \in I} e_{ \pm i}\left(\alpha_{ \pm j}^{-1} \alpha_{ \pm k}\left(e_{ \pm k} x e_{ \pm k}\right)\right) e_{ \pm i} \\
& =w^{*}-\lim _{j} w^{*}-\sum_{k \in I} \delta_{j, k} e_{ \pm i} x e_{ \pm i} \\
& =e_{ \pm i} x e_{ \pm i}
\end{aligned}
$$

Then since the multiplication in $\mathfrak{A}$ is jointly SOT-SOT continuous on bounded sets and hence jointly SOT- $w^{*}$ continuous on bounded sets 


$$
\begin{aligned}
w^{*}-\lim _{i} e_{ \pm i}\left(\Psi_{ \pm} \Theta_{ \pm}(x)\right) e_{ \pm i} & =w^{*}-\lim _{i} e_{ \pm i} x e_{ \pm i} \\
\left(\mathrm{SOT}-\lim _{i} e_{ \pm i}\right)\left(\Psi_{ \pm} \Theta_{ \pm}(x)\right)\left(\mathrm{SOT}-\lim _{i} e_{ \pm i}\right) & =\left(\mathrm{SOT}-\lim _{i} e_{ \pm i}\right) x\left(\mathrm{SOT}-\lim _{i} e_{ \pm i}\right) \\
\Psi_{ \pm} \Theta_{ \pm}(x) & =x
\end{aligned}
$$

Hence the result.

For every $i \in I$, take the compact $K_{ \pm i} \subseteq G$ s.t. $e_{ \pm i}=M \chi_{K_{ \pm i}}$ and $y_{ \pm i} \in G$ s.t. $\alpha_{ \pm i}=\operatorname{Ad}\left(L_{y_{ \pm}}\right)$. Then by property (iii) of Definition 5.2.1,

$$
y_{+j}^{-1} y_{+i} K_{+i} \cap y_{-j}^{-1} y_{-i} K_{-i}=\varnothing
$$

for $i, j, k, l \in I, i \neq j$ and $k \neq l$.

Consider the projections

$$
P_{ \pm}:=\bigvee_{i \neq j \in I} \alpha_{ \pm j}^{-1} \alpha_{ \pm i}\left(e_{ \pm i}\right)=M \chi_{Q_{ \pm}} \quad \text { where } \quad Q_{ \pm}=\bigcup_{i \neq j \in I} y_{ \pm j}^{-1} y_{ \pm i} K_{ \pm i}
$$

Proposition 5.3.4. $Q_{+}$and $Q_{-}$are disjoint and hence

$$
P_{+} \perp P_{-} \text {. }
$$

Proof.

$$
\begin{aligned}
Q_{+} \cap Q_{-} & =\left(\bigcup_{i \neq j \in I} y_{+j}^{-1} y_{+i} K_{+i}\right) \cap\left(\bigcup_{k \neq l \in I} y_{-l}^{-1} y_{-k} K_{-k}\right) \\
& =\bigcup_{i \neq j \in I} \bigcup_{k \neq l \in I} y_{+j}^{-1} y_{+i} K_{+i} \cap y_{-l}^{-1} y_{-k} K_{-k} \\
& =\bigcup_{i \neq j \in I} \bigcup_{k \neq l \in I} \varnothing \\
& =\varnothing .
\end{aligned}
$$


Lemma 5.3.5. $\alpha_{ \pm j}^{-1} \Theta_{ \pm j}(x)=P_{ \pm} \alpha_{ \pm j}^{-1} \Theta_{ \pm j}(x) P_{ \pm} \quad \forall x \in \mathfrak{A}, j \in I$.

Proof. First note that if $i \neq j$ then

$$
\alpha_{ \pm j}^{-1} \alpha_{ \pm i}\left(e_{ \pm i}\right) \leq \bigvee_{k \neq l \in I} \alpha_{ \pm l}^{-1} \alpha_{ \pm k}\left(e_{ \pm i}\right)=P_{ \pm} \quad \Rightarrow \quad P_{ \pm} \alpha_{ \pm j}^{-1} \alpha_{ \pm i}\left(e_{ \pm i}\right) P_{ \pm}=\alpha_{ \pm j}^{-1} \alpha_{ \pm i}\left(e_{ \pm i}\right)
$$

Now let $x \in \mathfrak{A}$.

$$
\begin{aligned}
\alpha_{ \pm j}^{-1} \Theta_{ \pm j}(x) & =\alpha_{ \pm j}^{-1} \sum_{j \neq i \in I} \alpha_{ \pm i}\left(e_{ \pm i} x e_{ \pm i}\right) \\
& =\sum_{j \neq i \in I} \alpha_{ \pm j}^{-1} \alpha_{ \pm i}\left(e_{ \pm i} x e_{ \pm i}\right) \\
& =\sum_{j \neq i \in I} \alpha_{ \pm j}^{-1} \alpha_{ \pm i}\left(e_{ \pm i}\right) \alpha_{ \pm j}^{-1} \alpha_{ \pm i}(x) \alpha_{ \pm j}^{-1} \alpha_{ \pm i}\left(e_{ \pm i}\right) \\
& =\sum_{j \neq i \in I} P_{ \pm} \alpha_{ \pm j}^{-1} \alpha_{ \pm i}\left(e_{ \pm i}\right) \alpha_{ \pm j}^{-1} \alpha_{ \pm i}(x) \alpha_{ \pm j}^{-1} \alpha_{ \pm i}\left(e_{ \pm i}\right) P_{ \pm} \\
& =\sum_{j \neq i \in I} P_{ \pm} \alpha_{ \pm j}^{-1} \alpha_{ \pm i}\left(e_{ \pm i} x e_{ \pm i}\right) P_{ \pm} \\
& =P_{ \pm} \alpha_{ \pm j}^{-1}\left(\sum_{j \neq i \in I} \alpha_{ \pm i}\left(e_{ \pm i} x e_{ \pm i}\right)\right) P_{ \pm} \\
& =P_{ \pm} \alpha_{ \pm j}^{-1} \Theta_{ \pm j}(x) P_{ \pm} .
\end{aligned}
$$

Proposition 5.3.6. For all $x, y \in \mathfrak{A}_{1}$,

$$
\left\|P_{+} x P_{+}+P_{-} y P_{-}\right\| \leq 1
$$


Proof. Let $x, y \in \mathfrak{A}_{1}$ and $f \in L_{p}(G)$. Since $Q_{+} \cap Q_{-}$,

$$
\begin{aligned}
& \left\|\left(P_{+} x P_{+}+P_{-} y P_{-}\right) f\right\|_{p}^{p} \\
= & \int_{G}\left|\left(P_{+} x P_{+} f+P_{-} y P_{-} f\right)^{p}\right| \\
= & \int_{G}\left|\left(\chi_{Q_{+}} x P_{+} f+\chi_{Q_{-}} y P_{-} f\right)^{p}\right| \\
= & \int_{G}\left|\left(\chi_{Q_{+}} x P_{+} f\right)^{p}+\left(\chi_{Q_{-}} y P_{-} f\right)^{p}\right| \\
= & \int_{Q_{+}}\left|x P_{+} f\right|^{p}+\int_{Q_{-}}\left|y P_{-} f\right|^{p} \\
\leq & \int_{G}\left|x P_{+} f\right|^{p}+\int_{G}\left|y P_{-} f\right|^{p} \\
= & \left\|x P_{+} f\right\|_{p}^{p}+\left\|y P_{-} f\right\|_{p}^{p} \\
\leq & \|x\|^{p}\left\|P_{+} f\right\|_{p}^{p}+\|y\|^{p}\left\|P_{-} f\right\|_{p}^{p} \\
\leq & \left\|P_{+} f\right\|_{p}^{p}+\left\|P_{-} f\right\|_{p}^{p} \\
= & \int_{G}\left|P_{+} f\right|^{p}+\int_{G}\left|P_{-} f\right|^{p} \\
= & \int_{G}\left|\chi_{Q_{+}} f\right|^{p}+\int_{G}\left|\chi_{Q_{-}} f\right|^{p} \\
= & \int_{Q_{+}}|f|^{p}+\int_{Q_{-}}|f|^{p} \\
= & \int_{Q_{+} \cup Q_{-}}|f|^{p} \\
\leq & \int_{G}|f|^{p} \\
= & \|f\|_{p}^{p} .
\end{aligned}
$$

Fix $\Phi \in B(\mathfrak{A})$ and $\rho \in \mathfrak{A}_{*}$ and define $\varphi, \varphi_{ \pm} \in \mathfrak{A}^{*}$ by

$$
\begin{gathered}
\varphi(x):=\langle\Phi(x), \rho\rangle \\
\varphi_{ \pm}(x):=\varphi\left(P_{ \pm} x P_{ \pm}\right)=\left\langle\Phi\left(P_{ \pm} x P_{ \pm}\right), \rho\right\rangle .
\end{gathered}
$$


Lemma 5.3.7. For $\varphi$ and $\varphi_{ \pm}$defined as above we have the inequality

$$
\left\|\varphi_{+}\right\|+\left\|\varphi_{-}\right\| \leq\|\varphi\|
$$

Proof. First note that for any Banach space $X$ and $f \in X^{*}$,

$$
\|f\|=\sup _{x \in X_{1}} \Re e f(x)
$$

(see p.39 of [13]). Then

$$
\begin{aligned}
\left\|\varphi_{+}\right\|+\left\|\varphi_{-}\right\| & =\sup _{x \in \mathfrak{R}_{1}} \Re \mathrm{e} \varphi_{+}(x)+\sup _{y \in \mathfrak{R}_{1}} \Re \operatorname{e} \varphi_{-}(y) \\
& =\sup _{x \in \mathfrak{R}_{1}} \Re \mathrm{e}\left\langle\Phi\left(P_{+} x P_{+}\right), \rho\right\rangle+\sup _{y \in \mathfrak{R}_{1}} \Re \mathrm{e}\left\langle\Phi\left(P_{-} y P_{-}\right), \rho\right\rangle \\
& =\sup _{x, y \in \mathfrak{A}_{1}} \Re \mathrm{e}\left\langle\Phi\left(P_{+} x P_{+}+P_{-} y P_{-}\right), \rho\right\rangle \\
& =\sup _{x, y \in \mathfrak{R}_{1}} \Re \mathrm{e} \varphi\left(P_{+} x P_{+}+P_{-} y P_{-}\right) \\
& \leq \sup _{w \in \mathfrak{A}_{1}} \Re \mathrm{e} \varphi(w) \quad \text { by Proposition } 5.3 .6 \\
& =\|\varphi\| .
\end{aligned}
$$

We are now ready to state and prove the main result of this chapter.

Theorem 5.3.8. Let $\left(\alpha_{ \pm i}, e_{ \pm i}\right)_{i \in I}$ be a compact spiral nebulae on $\mathfrak{A}=B\left(L_{p}(G)\right)$.

Let $\Phi \in B(\mathfrak{A})$ s.t. $\alpha_{ \pm i} \Phi=\Phi \alpha_{ \pm i}$ and $\alpha_{ \pm i}^{-1} \Phi=\Phi \alpha_{ \pm i}^{-1} \quad \forall i \in I$. Then if

$$
\Psi_{ \pm} \Phi=\Phi \Psi_{ \pm}
$$


where $\Psi_{ \pm}$are arbitrary (but fixed) $w^{*}$-cluster points of $\left(\alpha_{ \pm i}^{-1}\right)$ and $\forall i \in I$

$$
\Phi\left(e_{ \pm i} \mathfrak{A} e_{ \pm i}\right)=0
$$

then

$$
\Phi=0 .
$$

Proof. First let $x \in \mathfrak{A}$ and note that $\forall j \in I$

$$
\begin{aligned}
\Phi \Theta_{ \pm}(x) & =\Phi \sum_{i \in I} \alpha_{ \pm i}\left(e_{ \pm i} x e_{ \pm i}\right) \\
& =\Phi \alpha_{ \pm j}\left(e_{ \pm j} x e_{ \pm j}\right)+\Phi \sum_{j \neq i \in I} \alpha_{ \pm i}\left(e_{ \pm i} x e_{ \pm i}\right) \\
& =\Phi \alpha_{ \pm j}\left(e_{ \pm j} x e_{ \pm j}\right)+\Phi \Theta_{ \pm j}(x) \\
& =\alpha_{ \pm j} \Phi\left(e_{ \pm j} x e_{ \pm j}\right)+\Phi \Theta_{ \pm j}(x) \\
& =0+\Phi \Theta_{ \pm j}(x) \\
& =\Phi \Theta_{ \pm j}(x) .
\end{aligned}
$$

Now we calculate

$$
\begin{aligned}
\left\langle\alpha_{ \pm j}^{-1} \Phi \Theta_{ \pm}(x), \rho\right\rangle & =\left\langle\alpha_{ \pm j}^{-1} \Phi \Theta_{ \pm j}(x), \rho\right\rangle \\
& =\left\langle\Phi \alpha_{ \pm j}^{-1} \Theta_{ \pm j}(x), \rho\right\rangle \\
& =\left\langle\Phi\left(P_{ \pm} \alpha_{ \pm j}^{-1}\left(\Theta_{ \pm j}(x)\right) P_{ \pm}\right), \rho\right\rangle \quad \text { by Lemma } 5.3 .5 \\
& =\varphi_{ \pm}\left(\alpha_{ \pm i}^{-1} \Theta_{ \pm j}(x)\right)
\end{aligned}
$$


and hence

$$
\begin{aligned}
\left|\left\langle\alpha_{ \pm j}^{-1} \Phi \Theta_{ \pm j}(x), \rho\right\rangle\right| & \leq\left\|\varphi_{ \pm}\left(\alpha_{ \pm j}^{-1} \Theta_{ \pm j}(x)\right)\right\| \\
& \leq\left\|\varphi_{ \pm}\right\|\left\|\alpha_{ \pm j}^{-1}\right\|\left\|\Theta_{ \pm j}\right\|\|x\| \\
& \leq\left\|\varphi_{ \pm}\right\|\|x\| \quad \text { by Lemma 5.3.2. }
\end{aligned}
$$

Then if we write the cluster points

$$
\Psi_{ \pm}=w^{*}-\lim _{ \pm j} \alpha_{ \pm j}^{-1}
$$

for subnets $\left(\alpha_{ \pm j}^{-1}\right)$ of $\left(\alpha_{ \pm i}^{-1}\right)$ then

$$
\begin{aligned}
\left\|\left\langle\Phi \Psi_{ \pm} \Theta_{ \pm}(\cdot), \rho\right\rangle\right\| & =\sup _{x \in \mathfrak{A}_{1}}\left|\left\langle\Phi \Psi_{ \pm} \Theta_{ \pm}(x), \rho\right\rangle\right| \\
& =\sup _{x \in \mathfrak{A}_{1}}\left|\left\langle\Psi_{ \pm} \Phi \Theta_{ \pm}(x), \rho\right\rangle\right| \\
& =\sup _{x \in \mathfrak{A}_{1}}\left|\left\langle\left(w^{*}-\lim _{j} \alpha_{ \pm j}^{-1}\right) \Phi \Theta_{ \pm}(x), \rho\right\rangle\right| \\
& =\sup _{x \in \mathfrak{A}_{1}}\left|\left\langle w^{*}-\lim _{j}\left(\alpha_{ \pm j}^{-1} \Phi \Theta_{ \pm}(x)\right), \rho\right\rangle\right| \\
& =\sup _{x \in \mathfrak{A}_{1}} \lim _{j}\left|\left\langle\alpha_{ \pm j}^{-1} \Phi \Theta_{ \pm}(x), \rho\right\rangle\right| \\
& \leq \sup _{x \in \mathfrak{A}_{1}} \lim _{j}\left\|\varphi_{ \pm}\right\|\|x\| \\
& =\left\|\varphi_{ \pm}\right\| .
\end{aligned}
$$

Now since $\Psi_{ \pm} \Theta_{ \pm}=\mathrm{id}_{\mathfrak{A}}$ by Lemma 5.3.3,

$$
\begin{aligned}
\varphi(x) & =\langle\Phi(x), \rho\rangle=\frac{1}{2}\langle\Phi(x), \rho\rangle+\frac{1}{2}\langle\Phi(x), \rho\rangle=\frac{1}{2}\left\langle\Phi \Psi_{+} \Theta_{+}(x), \rho\right\rangle+\frac{1}{2}\left\langle\Phi \Psi_{-} \Theta_{-}(x), \rho\right\rangle \\
& \Rightarrow\|\varphi\|=\left\|\frac{1}{2}\left\langle\Phi \Psi_{+} \Theta_{+}, \rho\right\rangle+\frac{1}{2}\left\langle\Phi \Psi_{-} \Theta_{-}, \rho\right\rangle\right\| \leq \frac{1}{2}\left\|\varphi_{+}\right\|+\frac{1}{2}\left\|\varphi_{-}\right\| \leq \frac{1}{2}\|\varphi\|
\end{aligned}
$$

by Lemma 5.3.7, which forces $\varphi=0$, and then since $\rho \in \mathfrak{A}_{*}$ was arbitrary, $\Phi=0$. 


\section{Chapter 6}

\section{DTC Sets for Neufang's Algebra}

We are finally ready to prove the promised results. First we summarize what is known about the topological centres of $\mathcal{N}\left(\ell_{p}(G)\right)^{* *}$. Then we show that the left topological centre condition for $\mathcal{N}\left(\ell_{p}(G)\right)^{* *}$ is equivalent to the commutation of two representations. This allows us to apply Theorem 5.3.8 to prove the left strong Arens irregularity of $\mathcal{N}\left(\ell_{p}(G)\right)$ and produce a 2-point left DTC set if $G$ is infinite abelian discrete. We introduce a new notion of being determining for the topological centre in terms of representations which allows us to say that this set is left DTC (in this new sense) for $\mathcal{N}\left(\ell_{p}(G)\right)$ for nonabelian $G$. We are able to lift right DTC sets for $\ell_{1}(G)$ to $\mathcal{N}\left(\ell_{p}(G)\right)$ even for nonabelian discrete $G$.

Throughout this chapter, $p \in(1, \infty)$. As in Chapter 5 , the symbol " \pm " is meant to be replaced by either + or - , consistently. 


\subsection{The topological centres of $\mathcal{N}\left(L_{p}(G)\right)^{* *}$}

We now consider the second dual of $\mathcal{N}\left(L_{p}(G)\right)$ and its topological centres. First note that the module actions of $\mathcal{N}\left(L_{p}(G)\right)$ on $B\left(L_{p}(G)\right), \odot$ and $\cdot$, are exactly $\square$ and $\diamond$.

Theorem 6.1.1 (2.1 in [28]). Let $G$ be a second countable locally compact noncompact group. Then $\mathcal{N}\left(L_{p}(G)\right.$ ) is LSAI (left strongly Arens irregular) but not RSAI (right SAI), since

$$
M_{L_{\infty}(G)}^{\perp} \subset \mathcal{Z}_{t}^{2}\left(\mathcal{N}\left(L_{p}(G)\right)^{* *}\right) \quad \text { and } \quad M_{L_{\infty}(G)}^{\perp} \backslash \mathcal{N}\left(L_{p}(G)\right) \neq \varnothing
$$

This was the first such example.

We will prove that $\mathcal{N}\left(L_{p}(G)\right)$ is LSAI if $G$ is infinite discrete, we do not need the assumption that $G$ is second countable.

If $G$ is discrete, we can say a lot more. First of all, we can decompose $\mathcal{N}\left(\ell_{p}(G)\right)$ as follows.

Lemma 6.1.2 (Proposition 3.3.3 in [3]). Let $G$ be a discrete group. Then

$$
\left(\mathcal{N}\left(\ell_{p}(G)\right), *\right)=\left(\ell_{1}(G) \oplus M_{\ell_{\infty}(G)_{\perp}}, \circledast\right)
$$

as Banach algebras, where the product $\circledast$ is given by

$$
(f, \alpha) \circledast(g, \beta)=(f * g, f * \beta+\alpha * g)
$$

and $f \in \ell_{1}(G)$ is identified with

$$
\sum_{t \in G} f(t)\left(\delta_{t} \otimes \delta_{t}\right) \in \mathcal{N}\left(\ell_{p}(G)\right)
$$


This identification plays nicely with second duals:

\section{Lemma 6.1.3.}

$$
\left(\mathcal{N}\left(\ell_{p}(G)\right)^{* *}, \square\right)=\left(\ell_{1}(G)^{* *} \oplus M_{\ell_{\infty}(G)}^{\perp}, \mathbf{\beta}\right)
$$

and

$$
\left(\mathcal{N}\left(\ell_{p}(G)\right)^{* *}, \diamond\right)=\left(\ell_{1}(G)^{* *} \oplus M_{\ell_{\infty}(G)}^{\perp}, \mathbf{\phi}\right)
$$

as Banach algebras where and are given by

$$
(h, \Xi) \boldsymbol{\$}(k, \Upsilon)=(h \diamond k, h \square \Upsilon+\Xi \square k)
$$

and

$$
(h, \Xi)(k, \Upsilon)=(h \diamond k, h \diamond \Upsilon+\Xi \diamond k) .
$$

for $h, k \in \ell_{1}(G)$ and $\Xi, \Upsilon \in M_{\ell_{\infty}(G)}^{\perp}$.

Proof. By Proposition 3.3.4 in [3],

$$
\left(\mathcal{N}\left(\ell_{p}(G)\right)^{* *}, \diamond\right)=\left(\ell_{1}(G)^{* *} \oplus\left(M_{\ell_{\infty}(G)_{\perp}}\right)^{* *}, \mathbf{\phi}\right)
$$

and an easy modification of the proof yields that as well

$$
\left(\mathcal{N}\left(\ell_{p}(G)\right)^{* *}, \square\right)=\left(\ell_{1}(G)^{* *} \oplus\left(M_{\ell_{\infty}(G)_{\perp}}\right)^{* *}, \mathbf{\beta}\right) \text {. }
$$

It is proved in Theorem 3.3.2 in [3] that

$$
\left(M_{\ell_{\infty}(G)_{\perp}}\right)^{* *}=M_{\ell_{\infty}(G)}^{\perp}
$$


These identifications allow us to describe exactly the right topological centre of $\mathcal{N}\left(\ell_{p}(G)\right)^{* *}$.

Theorem 6.1.4 (3.2.2 in [3]). Let $G$ be infinite discrete. Then

$$
\mathcal{Z}_{t}^{2}\left(\mathcal{N}\left(\ell_{p}(G)\right)^{* *}\right)=\ell_{1}(G) \oplus M_{\ell_{\infty}(G)}^{\perp}
$$

This is one of the main results of [3].

Remark 6.1.5. By Proposition 5.3(i) in [9], $\left(\mathcal{N}\left(\ell_{p}(G)\right), \circ\right)$ is Arens regular, as is $\ell_{1}(G)$ with pointwise multiplication o by $\S 1.4 .8$ in $[31]$. So $\left(\mathcal{N}\left(L_{p}(G)\right), *\right)$ can be thought of as a quantized $\left(L_{1}(G), *\right)$ while $\left(\mathcal{N}\left(\ell_{p}(G)\right), \circ\right)$ is a quantized $\left(\ell_{1}(G), \circ\right)$.

\subsection{A representation of $\mathrm{LUC}(G)^{*}$}

Ghahramani showed in [15] that the map

$$
\gamma: M(G) \hookrightarrow B\left(B\left(L_{2}(G)\right)\right)
$$

given by for $\mu \in M(G)$ and $T \in B\left(L_{2}(G)\right)$,

$$
\gamma(\mu) T=\int_{G}^{w^{*}} L_{t^{-1}} T L_{t} d \mu(t)
$$

is an isometric algebra homomorphism. This was greatly extended by Neufang:

Proposition 6.2.1 (Satz 2.2.1 in [30]). The map

$$
\gamma: M(G) \hookrightarrow B^{\sigma}\left(B\left(L_{p}(G)\right)\right)
$$


given by for $\mu \in M(G)$ and $T \in B\left(L_{p}(G)\right)$

$$
\gamma(\mu) T=\int_{G}^{w^{*}} L_{t^{-1}} T L_{t} d \mu(t)
$$

is a $w^{*}$-w*-continuous isometric algebra homomorphisms which extends to a $w^{*}-w^{*}$ continuous isometry

$$
\widetilde{\gamma}: \operatorname{LUC}(G)^{*} \hookrightarrow B\left(B\left(L_{p}(G)\right)\right)
$$

given by for $f \in \operatorname{LUC}(G)^{*}$ and $T \in B\left(L_{p}(G)\right)$

$$
\langle\widetilde{\gamma}(f) T, \rho\rangle=\left\langle f,\left\langle L_{t^{-1}} T L_{t}, \rho\right\rangle\right\rangle
$$

The representation of $M(G)$ commutes with the representation $\Gamma$ of $X\left(L_{p}(G)\right)^{*}$ defined in Theorem 4.3.5. To prove this, we need the following result.

Proposition 6.2.2. Let $\rho \in \mathcal{N}\left(L_{p}(G)\right)$. Then the map

$$
G \ni t \mapsto L_{t} \rho L_{t^{-1}} \in \mathcal{N}\left(L_{p}(G)\right)
$$

is continuous.

Note that since the norm on $\mathcal{N}\left(L_{p}(G)\right)$ is different than the norm on $B\left(L_{p}(G)\right)$, this is not a consequence of Proposition 4.3.2.

Proof. Let $\xi \otimes \eta \in L_{q}(G) \otimes_{\pi} L_{p}(G)=\mathcal{N}\left(L_{p}(G)\right)$ be a rank-one operator. Then

$$
\left\|L_{t}(\xi \otimes \eta) L_{t^{-1}}-\xi \otimes \eta\right\|_{\mathcal{N}} \rightarrow 0 \quad \text { as } \quad t \rightarrow e
$$

follows by the same argument as in Proposition 4.3.2, replacing operator norms with 
nuclear norms. Now let $\rho \in \mathcal{N}\left(L_{p}(G)\right)$ and $\varepsilon>0$. Let

$$
\rho=\sum_{n=1}^{\infty} \xi_{n} \otimes \eta_{n}
$$

Then $\exists N \in \mathbb{N}$ s.t.

$$
\sum_{n=N+1}^{\infty}\left\|\xi_{n}\right\|_{q}\left\|\eta_{n}\right\|_{p}<\frac{\varepsilon}{4}
$$

For $i=1, \ldots, N$, let $U_{i}$ be a neighbourhood of $e \in G$ s.t. $\forall t \in U_{i}$

$$
\left\|L_{t^{-1}}\left(\xi_{n} \otimes \eta_{n}\right) L_{t}-\xi_{n} \otimes \eta_{n}\right\|_{\mathcal{N}}<\frac{\varepsilon}{2 N}
$$

Then $U:=\bigcap_{i=1}^{n} U_{i}$ is a neighbourhood of $e$ s.t. $\forall t \in U$

$$
\begin{aligned}
& \left\|L_{t} \rho L_{t^{-1}}-\rho\right\|_{\mathcal{N}} \\
= & \left\|L_{t}\left(\sum_{n=1}^{\infty} \xi_{n} \otimes \eta_{n}\right) L_{t^{-1}}-\sum_{n=1}^{\infty} \xi_{n} \otimes \eta_{n}\right\|_{\mathcal{N}} \\
\leq & \sum_{n=1}^{\infty}\left\|L_{t}\left(\xi_{n} \otimes \eta_{n}\right) L_{t^{-1}}-\xi_{n} \otimes \eta_{n}\right\|_{\mathcal{N}} \\
= & \sum_{n=1}^{N}\left\|L_{t}\left(\xi_{n} \otimes \eta_{n}\right) L_{t^{-1}}-\xi_{n} \otimes \eta_{n}\right\|_{\mathcal{N}}+\sum_{n=N+1}^{\infty}\left\|L_{t}\left(\xi_{n} \otimes \eta_{n}\right) L_{t^{-1}}-\xi_{n} \otimes \eta_{n}\right\|_{\mathcal{N}} \\
= & \sum_{n=1}^{N}\left\|L_{t}\left(\xi_{n} \otimes \eta_{n}\right) L_{t^{-1}}-\xi_{n} \otimes \eta_{n}\right\|_{\mathcal{N}}+\sum_{n=N+1}^{\infty}\left\|\left(L_{t} \xi_{n}\right) \otimes\left(L_{t} \eta_{n}\right)\right\|_{\mathcal{N}}+\sum_{n=N+1}^{\infty}\left\|\xi_{n} \otimes \eta_{n}\right\|_{\mathcal{N}} \\
\leq & \sum_{n=1}^{N}\left\|L_{t}\left(\xi_{n} \otimes \eta_{n}\right) L_{t^{-1}}-\xi_{n} \otimes \eta_{n}\right\|_{\mathcal{N}}+2 \sum_{n=N+1}^{\infty}\left\|\xi_{n}\right\|_{q}\left\|\eta_{n}\right\|_{p} \\
< & \sum_{n=1}^{N} \frac{\varepsilon}{2 N}+2 \frac{\varepsilon}{4} \\
= & \frac{\varepsilon}{2}+\frac{\varepsilon}{2} \\
= & \varepsilon .
\end{aligned}
$$


Lemma 6.2.3. $\Gamma$ and $\gamma$ commute, that is, $\forall m \in X\left(L_{p}(G)\right)^{*}$ and $\mu \in M(G)$,

$$
\Gamma(m) \gamma(\mu)=\gamma(\mu) \Gamma(m)
$$

Proof. We extend Neufang's proof of this result for $p=2$ from [27]. Let $T \in B\left(L_{p}(G)\right)$ and $\rho, \tau \in \mathcal{N}\left(L_{p}(G)\right)$. By Proposition 3.1.2 in [3],

$$
\left(L_{t} T L_{t^{-1}}\right) \odot \rho=L_{t}(T \odot \rho) L_{t^{-1}}
$$

Then by Proposition 4.2.2,

$$
\begin{aligned}
\left\langle\left(L_{t} \rho L_{t^{-1}}\right) \cdot T, \tau\right\rangle & =\left\langle T, \tau *\left(L_{t} \rho L_{t^{-1}}\right)\right\rangle \\
& =\left\langle T \odot \tau, L_{t} \rho L_{t^{-1}}\right\rangle \\
& =\left\langle L_{t^{-1}}(T \odot \tau) L_{t}, \rho\right\rangle \\
& =\left\langle\left(L_{t^{-1}} T L_{t}\right) \odot \tau, \rho\right\rangle \\
& =\left\langle\left(L_{t^{-1}} T L_{t}\right), \tau * \rho\right\rangle \\
& =\left\langle\rho \cdot\left(L_{t^{-1}} T L_{t}\right), \tau\right\rangle
\end{aligned}
$$

Hence

$$
\left(L_{t} \rho L_{t^{-1}}\right) \cdot T=\rho \cdot\left(L_{t^{-1}} T L_{t}\right)
$$

By Proposition 6.2.2 the map

$$
G \ni t \mapsto\left(L_{t} \rho L_{t^{-1}}\right) \cdot T \in X\left(L_{p}(G)\right)
$$

is continuous. 
Since it is also bounded, by Proposition 2.1.8, the weak integral

$$
\int_{G}^{w}\left(L_{t} \rho L_{t^{-1}}\right) \cdot T d \mu(t)
$$

exists $\forall \mu \in M(G)$.

Now let $m \in X\left(L_{p}(G)\right)^{*}, \mu \in M(G), T \in B\left(L_{p}(G)\right)$, and $\rho \in \mathcal{N}\left(\ell_{p}(G)\right)$.

$$
\begin{aligned}
\langle\gamma(\mu) \Gamma(m) T, \rho\rangle & =\int_{G}\left\langle L_{t^{-1}} \Gamma(m) T L_{t}, \rho\right\rangle d \mu(t) \\
& =\int_{G}\left\langle\Gamma(m) T, L_{t} \rho L_{t^{-1}}\right\rangle d \mu(t) \\
& =\int_{G}\left\langle m,\left(L_{t} \rho L_{t^{-1}}\right) \cdot T\right\rangle d \mu(t) \\
& =\int_{G}\left\langle m, \rho \cdot\left(L_{t^{-1}} T L_{t}\right)\right\rangle d \mu(t) \\
& =\left\langle m, \int_{G}^{w} \rho \cdot\left(L_{t^{-1}} T L_{t}\right) d \mu(t)\right\rangle \\
& =\left\langle m, \int_{G}^{w^{*}} \rho \cdot\left(L_{t^{-1}} T L_{t}\right) d \mu(t)\right\rangle \\
& =\left\langle m, \int_{G}^{w^{*}} \int_{G}^{w^{*}} L_{s^{-1}} L_{t^{-1}} T L_{t} L_{s} \pi(\rho)(s) d s d \mu(t)\right\rangle \\
& =\left\langle m, \int_{G}^{w^{*}} \int_{G}^{w^{*}} L_{(s t)^{-1}} T L_{s t} \pi(\rho)(s) d s d \mu(t)\right\rangle \\
& =\left\langle m, \int_{G}^{w^{*}} L_{t^{-1}} T L_{t} d(\pi(\rho) * \mu)(t)\right\rangle \\
& =\langle m, \gamma(\pi(\rho * \mu)) T\rangle .
\end{aligned}
$$

On the other hand,

$$
\langle\Gamma(m) \gamma(\mu) T, \rho\rangle=\langle m, \rho \cdot \gamma(\mu) T\rangle=\langle m, \gamma(\pi(\rho)) \gamma(\mu) T\rangle=\langle m, \gamma(\pi(\rho) * \mu) T\rangle .
$$

In the next section, we will show that $\Gamma$ commuting with $\tilde{\gamma}$ is equivalent to the left topological centre condition for $\mathcal{N}\left(\ell_{p}(G)\right)^{* *}$. 
Remark 6.2.4. We can realize the action of $\tilde{\gamma}$ as a left Arens module action in the following way. Let $f \in \operatorname{LUC}(G)^{*} \supset M(G)$. By Proposition 2.2.2 in [30],

$$
\operatorname{LUC}(G)=\left\{\left\langle L_{t^{-1}} T L_{t}, \xi \otimes \eta\right\rangle: T \in B\left(L_{p}(G)\right), \xi \in L_{q}(G), \eta \in L_{p}(G)\right\}
$$

Define $f^{\prime} \in M_{\mathrm{LUC}(G)}^{*}$ via for $T \in B\left(L_{p}(G)\right)$ and $\rho \in \mathcal{N}\left(L_{p}(G)\right)$

$$
\left\langle f^{\prime}, T \odot \rho\right\rangle=\left\langle f^{\prime}, M_{\left\langle L_{t} T L_{t^{-1}}, \rho\right\rangle}\right\rangle:=\left\langle f \overline{\left\langle L_{t} T L_{t^{-1}}, \rho\right\rangle}\right\rangle=\left\langle f,\left\langle L_{t^{-1}} T L_{t}, \rho\right\rangle\right\rangle=\langle\tilde{\gamma}(f) T, \rho\rangle .
$$

Then let $\hat{f}$ be a Hahn-Banach extension of $f^{\prime}$ to $B\left(L_{p}(G)\right)$. Now

$$
\langle\tilde{\gamma}(f) T, \rho\rangle=\left\langle f^{\prime}, T \odot \rho\right\rangle=\langle\hat{f}, T \odot \rho\rangle=\langle\hat{f}, T \square \rho\rangle=\langle\hat{f} \square T, \rho\rangle .
$$

Remark 6.2.5. If $G$ is discrete and $f \in \ell_{1}(G)=M(G) \subset \operatorname{LUC}(G)^{*}$, then we can take

$$
\hat{f}=(f, 0) \in \ell_{1}(G) \oplus M_{\ell_{\infty}(G)_{\perp}} \subset \ell_{1}(G)^{* *} \oplus M_{\ell_{\infty}(G)}^{\perp}=\mathcal{N}\left(\ell_{p}(G)\right)^{* *} .
$$

This particular choice of $\hat{f}$ is indeed an extension of $f^{\prime}$, since for $h \in \operatorname{LUC}(G)^{*}$,

$$
\begin{aligned}
\left\langle\hat{f}, M_{h}\right\rangle & =\left\langle M_{h},(f, 0)\right\rangle \\
& =\left\langle M_{h}, \sum_{t \in G} f(t)\left(\delta_{t} \otimes \delta_{t}\right)\right\rangle \\
& =\sum_{t \in G} f(t)\left\langle\delta_{t}, h \delta_{t}\right\rangle \\
& =\sum_{t \in G} f(t) \sum_{s \in G} \delta_{t}(s)\left(h \delta_{t}\right)(s) \\
& =\sum_{t \in G} h(t) f(t) \\
& =\langle f, h\rangle .
\end{aligned}
$$


For $f \in \ell_{1}(G)$ we always take $\hat{f}$ as above. Note that with this convention

$$
\widehat{\ell_{1}(G)}=\widehat{M(G)} \subset \mathcal{Z}_{t}^{1}\left(\mathcal{N}\left(\ell_{p}(G)\right)^{* *}\right)
$$

Remark 6.2.6. The module action - of $\mathcal{N}\left(L_{p}(G)\right)$ on $B\left(L_{p}(G)\right)$, $\gamma$, and the left Arens module action $\square$ of $\mathcal{N}\left(L_{p}(G)\right)^{* *}$ on $B\left(L_{p}(G)\right)$ are related by the following identity: $\forall \rho \in \mathcal{N}\left(L_{p}(G)\right)$ and $T \in B\left(L_{p}(G)\right)$

$$
\rho \cdot T=\gamma(\pi(\rho)) T=\widehat{\pi(\rho)} \square T
$$

\subsection{The left centre condition and commuting}

\section{representations}

In this section we translate the left topological centre condition on $\mathcal{N}\left(\ell_{p}(G)\right)^{* *}$ to a commutation condition.

First note that if $G$ is discrete then $X\left(\ell_{p}(G)\right)=B\left(\ell_{p}(G)\right)$ and hence

$$
X\left(\ell_{p}(G)\right)^{*}=B\left(\ell_{p}(G)\right)^{*}=\mathcal{N}\left(\ell_{p}(G)\right)^{* *}
$$

So we can think of $\Gamma$ as an antirepresentation of $\mathcal{N}\left(\ell_{p}(G)\right)^{* *}$.

Lemma 6.3.1. Let $G$ be a discrete group, $m \in \mathcal{N}\left(\ell_{p}(G)\right)^{* *}=X\left(\ell_{p}(G)\right)^{*}, T \in B\left(\ell_{p}(G)\right)$, and $\rho \in \mathcal{N}\left(\ell_{p}(G)\right)$. Also let $f \in \operatorname{LUC}(G)^{*}$ and write $f=w^{*}-\lim _{\alpha} \mu_{\alpha}$ with the $\mu_{\alpha} \in M(G)$. Then

$$
\begin{aligned}
& \langle\Gamma(m) \tilde{\gamma}(f) T, \rho\rangle=\langle\tilde{\gamma}(f) \Gamma(m) T, \rho\rangle \quad \forall T \in B\left(\ell_{p}(G)\right) \\
\Longleftrightarrow & m \square w^{*}-\lim _{\alpha} \widehat{\pi(\rho)} \square \widehat{\mu_{\alpha}}=w^{*}-\lim _{\alpha} m \square \widehat{\pi(\rho)} \square \widehat{\mu_{\alpha}} \quad \forall \rho \in \mathcal{N}\left(\ell_{p}(G)\right) .
\end{aligned}
$$


Proof.

$$
\begin{aligned}
\langle\Gamma(m) \tilde{\gamma}(f) T, \rho\rangle & =\langle m, \rho \cdot \tilde{\gamma}(f) T\rangle \\
& =\left\langle m, \rho \cdot \tilde{\gamma}\left(w^{*}-\lim _{\alpha} \mu_{\alpha}\right) T\right\rangle \\
& =\left\langle m, \rho \cdot\left(w^{*}-\lim _{\alpha} \gamma\left(\mu_{\alpha}\right)\right) T\right\rangle \\
& =\left\langle m, \gamma(\pi(\rho))\left(w^{*}-\lim _{\alpha} \gamma\left(\mu_{\alpha}\right)\right) T\right\rangle \\
& =\left\langle m, w^{*}-\lim _{\alpha} \gamma(\pi(\rho)) \gamma\left(\mu_{\alpha}\right) T\right\rangle \\
& =\left\langle m, w^{*}-\lim _{\alpha} \widehat{\pi(\rho)} \square \widehat{\mu_{\alpha}} \square T\right\rangle \\
& =\left\langle m,\left(w^{*}-\lim _{\alpha} \widehat{\pi(\rho)} \square \widehat{\mu_{\alpha}}\right) \square T\right\rangle \\
& =\left\langle m \square w^{*}-\lim _{\alpha} \widehat{\pi(\rho)} \square \widehat{\mu_{\alpha}}, T\right\rangle
\end{aligned}
$$

and

$$
\begin{aligned}
\langle\tilde{\gamma}(f) \Gamma(m) T, \rho\rangle & =\left\langle\tilde{\gamma}\left(w^{*}-\lim _{\alpha} \mu_{\alpha}\right) \Gamma(m) T, \rho\right\rangle \\
& =\left\langle w^{*}-\lim _{\alpha} \gamma\left(\mu_{\alpha}\right) \Gamma(m) T, \rho\right\rangle \\
& =\lim _{\alpha}\left\langle\gamma\left(\mu_{\alpha}\right) \Gamma(m) T, \rho\right\rangle \\
& =\lim _{\alpha}\left\langle\Gamma(m) \gamma\left(\mu_{\alpha}\right) T, \rho\right\rangle \quad \text { by Lemma } 6.2 .3 \\
& =\lim _{\alpha}\left\langle m, \rho \cdot \gamma\left(\mu_{\alpha}\right) T\right\rangle \\
& =\lim _{\alpha}\left\langle m, \gamma(\pi(\rho)) \gamma\left(\mu_{\alpha}\right) T\right\rangle \\
& =\lim _{\alpha}\left\langle m, \gamma\left(\pi(\rho) * \mu_{\alpha}\right) \square T\right\rangle \\
& =\lim _{\alpha}\left\langle m, \widehat{\pi(\rho)} \square \widehat{\mu_{\alpha}} \square T\right\rangle \\
& =\lim _{\alpha}\left\langle m \square \widehat{\pi(\rho)} \square \widehat{\mu_{\alpha}}, T\right\rangle \\
& =\left\langle w^{*}-\lim _{\alpha} \square \widehat{\pi(\rho)} \square \widehat{\mu_{\alpha}}, T\right\rangle .
\end{aligned}
$$

This result yields that the left topological centre condition on $\mathcal{N}\left(\ell_{p}(G)\right)^{* *}$ is equivalent to a commutation condition on $\Gamma$ and $\tilde{\gamma}$. 
Theorem 6.3.2. Let $G$ be discrete. Then $m \in \mathcal{Z}_{t}^{1}\left(\mathcal{N}\left(\ell_{p}(G)\right)^{* *}\right)$ iff

$$
\Gamma(m) \tilde{\gamma}(f)=\tilde{\gamma}(f) \Gamma(m)
$$

$\forall f \in \operatorname{LUC}(G)^{*}$.

Proof. First suppose that $m \in \mathcal{Z}_{t}^{1}\left(\mathcal{N}\left(\ell_{p}(G)\right)^{* *}\right)$. Then $\forall \rho \in \mathcal{N}\left(\ell_{p}(G)\right), f \in \operatorname{LUC}(G)^{*}$, and nets $\left(\mu_{\alpha}\right)$ in $M(G)$ s.t. $f=w^{*}-\lim \mu_{\alpha}$,

$$
m \square w^{*}-\lim _{\alpha} \widehat{\pi(\rho)} \square \widehat{\mu_{\alpha}}=w^{*}-\lim _{\alpha} m \square \widehat{\pi(\rho)} \square \widehat{\mu_{\alpha}}
$$

so by Lemma 6.3.1,

$$
\Gamma(m) \tilde{\gamma}(f)=\tilde{\gamma}(f) \Gamma(m)
$$

Now suppose that $\forall f \in \operatorname{LUC}(G)^{*}$,

$$
\Gamma(m) \tilde{\gamma}(f)=\tilde{\gamma}(f) \Gamma(m)
$$

Let $n \in \mathcal{N}\left(\ell_{p}(G)\right)^{* *}$ and define $f \in \operatorname{LUC}(G)^{*}$ by $f=\left.n\right|_{M_{\mathrm{LUC}(G)}}$. Then

$$
\begin{aligned}
\langle n \square T, \rho\rangle & =\langle n, T \square \rho\rangle \\
& =\langle n, T \odot \rho\rangle \\
& =\left\langle n, M_{\left\langle L_{t} T L_{t^{-1}}, \rho\right\rangle}\right\rangle \\
& =\left\langle f, \overline{\left\langle L_{t^{-1}} T L_{t}, \rho\right\rangle}\right\rangle \\
& =\langle\widetilde{\gamma}(f) T, \rho\rangle
\end{aligned}
$$

and hence

$$
n \square T=\widetilde{\gamma}(f) T
$$


Let $\left(n_{\alpha}\right)$ be a $w^{*}$-convergent net in $\mathcal{N}\left(L_{p}(G)\right)^{* *}$ and for each $\alpha$ let $f_{\alpha}=\left.n_{\alpha}\right|_{M_{\mathrm{LUC}(G)}}$. Then if $\zeta \in \mathcal{N}\left(L_{p}(G)\right)$ s.t. $\pi(\zeta)=\delta_{e}$ then

$$
\begin{aligned}
\left\langle m \square w^{*}-\lim _{\alpha} n_{\alpha}, T\right\rangle & =\left\langle m,\left(w^{*}-\lim _{\alpha} n_{\alpha}\right) \square T\right\rangle \\
& =\left\langle m, w^{*}-\lim _{\alpha} n_{\alpha} \square T\right\rangle \\
& =\left\langle m, w^{*}-\lim _{\alpha} \widetilde{\gamma}\left(f_{\alpha}\right) T\right\rangle \\
& =\left\langle m,\left(w^{*}-\lim _{\alpha} \widetilde{\gamma}\left(f_{\alpha}\right)\right) T\right\rangle \\
& =\left\langle m, \widetilde{\gamma}\left(w^{*}-\lim _{\alpha} f_{\alpha}\right) T\right\rangle \\
& =\left\langle m, \zeta \cdot \widetilde{\gamma}\left(w^{*}-\lim _{\alpha} f_{\alpha}\right) T\right\rangle \\
& =\left\langle\Gamma(m) \widetilde{\gamma}\left(w^{*}-\lim _{\alpha} f_{\alpha}\right) T, \zeta\right\rangle
\end{aligned}
$$

and

$$
\begin{aligned}
\left\langle w^{*}-\lim _{\alpha} m \square n_{\alpha}, T\right\rangle & =\lim _{\alpha}\left\langle m \square n_{\alpha}, T\right\rangle \\
& =\lim _{\alpha}\left\langle m, n_{\alpha} \square T\right\rangle \\
& =\lim _{\alpha}\left\langle m, \widetilde{\gamma}\left(f_{\alpha}\right) T\right\rangle \\
& =\lim _{\alpha}\left\langle m, \zeta \cdot \widetilde{\gamma}\left(f_{\alpha}\right) T\right\rangle \\
& =\lim _{\alpha}\left\langle\Gamma(m) \widetilde{\gamma}\left(f_{\alpha}\right) T, \zeta\right\rangle \\
& =\lim _{\alpha}\left\langle\widetilde{\gamma}\left(f_{\alpha}\right) \Gamma(m) T, \zeta\right\rangle \\
& =\left\langle w^{*}-\lim _{\alpha} \widetilde{\gamma}\left(f_{\alpha}\right) \Gamma(m) T, \zeta\right\rangle \\
& =\left\langle\widetilde{\gamma}\left(w^{*}-\lim _{\alpha} f_{\alpha}\right) \Gamma(m) T, \zeta\right\rangle .
\end{aligned}
$$

Therefore

$$
m \square w^{*}-\lim _{\alpha} n_{\alpha}=w^{*}-\lim _{\alpha} m \square n_{\alpha}
$$

and hence $m \in \mathcal{Z}_{t}^{1}\left(\mathcal{N}\left(\ell_{p}(G)\right)^{* *}\right)$. 
This result, while philosophically important (see Definition 6.4.3), is not sufficient since for DTC results we need a commutation condition equivalent to multiplication by elements the second dual being $w^{*}-w^{*}$-continuous against individual nets. Unfortunately, the result one expects, that if $w^{*}-\lim _{\alpha} n_{\alpha}=n \in \mathcal{N}\left(\ell_{p}(G)\right)^{* *}$ then

$$
m \square w^{*}-\lim _{\alpha} n_{\alpha}=w^{*}-\lim _{\alpha} m \square n_{\alpha} \quad \Longleftrightarrow \quad \Gamma(m) \tilde{\gamma}\left(\left.n\right|_{\operatorname{LUC}(G)}\right)=\tilde{\gamma}\left(\left.n\right|_{\operatorname{LUC}(G)}\right) \Gamma(m)
$$

is not true for two subtle reasons which can be seen in Lemma 6.3.1. Firstly, the $\rho \in \mathcal{N}\left(\ell_{p}(G)\right)=B\left(\ell_{p}(G)\right)_{*}$ we take only to separate the points of $B\left(\ell_{p}(G)\right)$ interferes with the net, and secondly, we need to approximate $f \in \operatorname{LUC}(G)^{*}$ by a net of $\mu_{\alpha} \in M(G)$ in order to apply Lemma 6.2.3 - this corresponds to approximating $n$ by a net of the form $n_{\alpha}=\widehat{\mu_{\alpha}}$. Lemma 6.3 .1 is the best result we have in general. This motivates an alternate definition of DTC in terms of commuting representations which we will give in the following section. However, if $G$ is abelian, we can get around the first problem.

Theorem 6.3.3. Let $G$ be abelian discrete and $m \in \mathcal{N}\left(\ell_{p}(G)\right)^{* *}$. Then

$$
\Gamma(m) \tilde{\gamma}(f)=\tilde{\gamma}(f) \Gamma(m) \quad \Longleftrightarrow \quad m \square w^{*}-\lim _{\alpha} \widehat{\mu_{\alpha}}=w^{*}-\lim _{\alpha} m \square \widehat{\mu_{\alpha}}
$$

for every net $\left(\mu_{\alpha}\right)$ in $M(G)$ converging $w^{*}$ to $f \in \operatorname{LUC}(G)^{*}$.

Proof. First suppose that

$$
\Gamma(m) \tilde{\gamma}(f)=\tilde{\gamma}(f) \Gamma(m) .
$$

Let $f=w^{*}-\lim _{\alpha} \mu_{\alpha}$ with the $\mu_{\alpha} \in M(G)$. Take $\zeta \in \mathcal{N}\left(\ell_{p}(G)\right)$ s.t. $\pi(\zeta)=\delta_{e}$. 
Then $\forall T \in B\left(\ell_{p}(G)\right)$

$$
\widehat{\pi(\zeta)} \square T=\gamma(\pi(\zeta)) T=\gamma\left(\delta_{e}\right) T=\int_{G}^{w^{*}} L_{t^{-1}} T L_{t} d \delta_{e}(t)=L_{e^{-1}} T L_{e}=T
$$

Then by Lemma 6.4.1, in particular

$$
\begin{gathered}
m \square w^{*}-\lim _{\alpha} \widehat{\pi(\zeta)} \square \widehat{\mu_{\alpha}}=w^{*}-\lim _{\alpha} m \square \widehat{\pi(\zeta)} \square \widehat{\mu_{\alpha}} \\
m \square w^{*}-\lim _{\alpha} \widehat{\mu_{\alpha}}=w^{*}-\lim _{\alpha} m \square \widehat{\mu_{\alpha} .}
\end{gathered}
$$

Now suppose that $\forall f=w^{*}-\lim _{\alpha} \mu_{\alpha} \in \operatorname{LUC}(G)^{*}$ with the $\mu_{\alpha} \in M(G)$

$$
m \square w^{*}-\lim _{\alpha} \widehat{\mu_{\alpha}}=w^{*}-\lim _{\alpha} m \square \widehat{\mu_{\alpha}}
$$

Note that since $G$ is abelian, $M(G)$ is commutative, so $\forall \rho \in \mathcal{N}\left(\ell_{p}(G)\right), \mu \in M(G)$, and $T \in B\left(\ell_{p}(G)\right)$

$$
\begin{aligned}
\widehat{\pi(\rho)} \square \widehat{\mu} \square T & =\gamma(\pi(\rho)) \gamma(\mu) T \\
& =\gamma(\pi(\rho) * \mu) T \\
& =\gamma(\mu * \pi(\rho)) T \\
& =\gamma(\mu) \gamma(\pi(\rho)) T \\
& =\widehat{\mu} \square \widehat{\pi(\rho)} \square T .
\end{aligned}
$$


Then $\forall \rho \in \mathcal{N}\left(\ell_{p}(G)\right)$ and $T \in B\left(\ell_{p}(G)\right)$

$$
\begin{aligned}
\left\langle m \square w^{*}-\lim _{\alpha} \widehat{\pi(\rho)} \square \widehat{\mu_{\alpha}}, T\right\rangle & =\left\langle m,\left(w^{*}-\lim _{\alpha} \widehat{\pi(\rho)} \square \widehat{\mu_{\alpha}}\right) \square T\right\rangle \\
& =\left\langle m, w^{*}-\lim _{\alpha} \widehat{\pi(\rho)} \square \widehat{\mu_{\alpha}} \square T\right\rangle \\
& =\left\langle m, w^{*}-\lim _{\alpha} \widehat{\mu_{\alpha}} \square \widehat{\pi(\rho)} \square T\right\rangle \\
& =\left\langle m,\left(w^{*}-\lim _{\alpha} \widehat{\mu_{\alpha}}\right) \square \widehat{\pi(\rho)} \square T\right\rangle \\
& =\left\langle m \square w^{*}-\lim _{\alpha} \widehat{\mu_{\alpha}}, \widehat{\pi(\rho)} \square T\right\rangle \\
& =\left\langle w^{*}-\lim _{\alpha} m \square \widehat{\mu_{\alpha}}, \widehat{\pi(\rho)} \square T\right\rangle \\
& =\left\langle w^{*}-\lim _{\alpha} m, \widehat{\mu_{\alpha}} \square \widehat{\pi(\rho)} \square T\right\rangle \\
& =\left\langle w^{*}-\lim _{\alpha} m, \widehat{\pi(\rho)} \square \widehat{\mu_{\alpha}} \square T\right\rangle \\
& =\left\langle w^{*}-\lim _{\alpha} m \square \widehat{\pi(\rho)} \square \widehat{\mu_{\alpha}}, T\right\rangle .
\end{aligned}
$$

Hence

$$
m \square w^{*}-\lim _{\alpha} \widehat{\pi(\rho)} \square \widehat{\mu_{\alpha}}=w^{*}-\lim _{\alpha} m \square \widehat{\pi(\rho)} \square \widehat{\mu_{\alpha}}
$$

and then by Lemma 6.4.1,

$$
\Gamma(m) \tilde{\gamma}(f)=\tilde{\gamma}(f) \Gamma(m) .
$$

\subsection{Left strong Arens irregularity of $\mathcal{N}\left(\ell_{p}(G)\right)$ and left DTC sets}

Here we give the main results of this thesis. First we interpret Theorem 5.3.8 (the automatic normality result) in our context. In this section, all groups are infinite discrete, and hence non-compact. This was a hypothesis of Theorem 5.3.8. 
Lemma 6.4.1. Let $G$ be an infinite discrete group. There exist

$$
\psi_{+}, \psi_{-} \in{\overline{\delta_{G}}}^{\omega^{*}} \subseteq \mathrm{LUC}(G)^{*}
$$

s.t. if $m \in \mathcal{N}\left(\ell_{p}(G)\right)^{* *}$ satisfies

$$
\Gamma(m) \tilde{\gamma}\left(\psi_{ \pm}\right)=\tilde{\gamma}\left(\psi_{ \pm}\right) \Gamma(m)
$$

then $m \in \mathcal{N}\left(\ell_{p}(G)\right)$.

Proof. Let $\left(\alpha_{ \pm i}, e_{ \pm i}\right)_{i \in I}$ be a compact spiral nebula on $B\left(\ell_{p}(G)\right)$ (see Section 5.2).

Let $\left(\alpha_{+j}^{-1}\right)_{+j \in+J}$ and $\left(\alpha_{-j}^{-1}\right)_{-j \in-J}$ be subnets of $\left(\alpha_{+i}^{-1}\right)_{i \in I}$ and $\left(\alpha_{-i}^{-1}\right)_{i \in I}$ converging to arbitrary $w^{*}$-cluster points $\Psi_{+}$and $\Psi_{-}$of $\left(\alpha_{+i}\right)_{i \in I}$ and $\left(\alpha_{-i}\right)_{i \in I}$, respectively.

For every $\pm j \in \pm J$ take the $x_{ \pm j} \in G$ s.t. $\alpha_{ \pm j}=\operatorname{Ad}\left(L_{x_{ \pm j}}\right)$. Then $\forall T \in B\left(\ell_{p}(G)\right)$

$$
\gamma\left(\delta_{x_{ \pm j}^{-1}}\right) T=\int_{G} L_{t^{-1}} T L_{t} d \delta_{x_{ \pm j}^{-1}}(t)=L_{x_{ \pm j}} T L_{x_{ \pm j}^{-1}}=\operatorname{Ad}\left(L_{x_{ \pm j}}\right) T=\alpha_{ \pm j}(T)
$$

hence

$$
\alpha_{ \pm j}=\gamma\left(\delta_{x_{ \pm} j}^{-1}\right) \quad \text { and } \quad \alpha_{ \pm j}^{-1}=\gamma\left(\delta_{x_{ \pm j}}\right)
$$

Then since $\tilde{\gamma}$ is $w^{*}$ - $w^{*}$-continuous (Proposition 6.2.1),

$$
\Psi_{ \pm}=w^{*}-\lim _{ \pm j} \alpha_{ \pm j}^{-1}=w^{*}-\lim _{ \pm j} \gamma\left(\delta_{x_{ \pm j}}\right)=\tilde{\gamma}\left(w^{*}-\lim _{ \pm j} \delta_{x_{ \pm j}}\right)=\tilde{\gamma}\left(\psi_{ \pm}\right)
$$

with

$$
\psi_{ \pm}:=w^{*}-\lim _{ \pm j} \delta_{x_{ \pm j}} \in{\overline{\delta_{G}}}^{w^{*}} \subseteq \operatorname{LUC}(G)^{*}
$$

Now suppose that $m \in \mathcal{N}\left(L_{p}(G)\right)^{* *}$ satisfies $\Gamma(m) \tilde{\gamma}\left(\psi_{ \pm}\right)=\tilde{\gamma}\left(\psi_{ \pm}\right) \Gamma(m)$. 
Note that by the Dixmier projection (Proposition 2.1.6) we have

$$
\mathcal{N}\left(L_{p}(G)\right)^{* *}=\mathcal{K}\left(L_{p}(G)\right)^{* * *}=\mathcal{K}\left(L_{p}(G)\right)^{*} \oplus \mathcal{K}\left(L_{p}(G)\right)^{\perp}=\mathcal{N}\left(L_{p}(G)\right) \oplus \mathcal{K}\left(L_{p}(G)\right)^{\perp}
$$

So we can write $m=\tau+m_{s}$ with $\tau \in \mathcal{N}\left(\ell_{p}(G)\right)$ and $m_{s} \in \mathcal{K}\left(\ell_{p}(G)\right)^{\perp}$.

Since $\tau \in \mathcal{N}\left(\ell_{p}(G)\right) \subseteq \mathcal{Z}_{t}^{1}\left(\mathcal{N}\left(\ell_{p}(G)\right)^{* *}\right)$, by Theorem 6.3.2, $\Gamma(\tau)$ commutes with $\Psi_{ \pm}=\tilde{\gamma}\left(\psi_{ \pm}\right)$and since $\Gamma(m)$ commutes with the $\Psi_{ \pm}=\tilde{\gamma}\left(\psi_{ \pm}\right)$by assumption,

$$
\Gamma\left(m_{s}\right)=\Gamma(m)-\Gamma(\tau)
$$

commutes with $\Psi_{ \pm}$.

By Lemma 6.2.3, $\Phi=\Gamma\left(m_{s}\right)$ commutes with the $\gamma\left(\delta_{x_{ \pm j}}\right)=\alpha_{ \pm j}^{-1}$ and $\gamma\left(\delta_{x_{ \pm j}^{-1}}\right)=\alpha_{ \pm j}$. Let $i \in I$. Then $e_{ \pm i}=M \chi_{K}$ for some compact and hence finite $K \subseteq G$, so $e_{ \pm i}$ is of finite rank. Then $e_{ \pm i} T e_{ \pm i}$ is also of finite rank and hence compact so

$$
\begin{aligned}
\left\langle\Phi\left(e_{ \pm i} T e_{ \pm i}\right), \rho\right\rangle & =\left\langle\Gamma\left(m_{s}\right)\left(e_{ \pm i} T e_{ \pm i}\right), \rho\right\rangle \\
& =\left\langle m_{s}, \rho \cdot\left(e_{ \pm i} T e_{ \pm i}\right)\right\rangle \\
& =0
\end{aligned}
$$

since $m_{s} \in \mathcal{K}\left(\ell_{p}(G)\right)^{\perp}$ and $\rho \cdot\left(e_{ \pm j} T e_{ \pm j}\right) \in \mathcal{K}\left(\ell_{p}(G)\right)$ by Lemma 4.2.3.

Then by Theorem 5.3.8,

$$
\Phi=\Gamma\left(m_{s}\right)=0 \quad \Rightarrow \quad m_{s}=0
$$

since $\Gamma$ is isometric. Therefore

$$
m=\tau+m_{s}=\tau \in \mathcal{N}\left(\ell_{p}(G)\right)
$$


This result gives us both that $\mathcal{N}\left(\ell_{p}(G)\right)$ is LSAI and left DTC sets for $\mathcal{N}\left(\ell_{p}(G)\right)$.

Theorem 6.4.2. $\mathcal{N}\left(\ell_{p}(G)\right)$ is LSAI if $G$ is infinite and discrete.

Proof. Let $m \in \mathcal{Z}_{t}^{1}\left(\mathcal{N}\left(\ell_{p}(G)\right)^{* *}\right)$. By Theorem 6.3.2, $\Gamma(m) \tilde{\gamma}\left(\psi_{ \pm}\right)=\tilde{\gamma}\left(\psi_{ \pm}\right) \Gamma(m)$.

Then by Lemma 6.4.1, $m \in \mathcal{N}\left(\ell_{p}(G)\right)$. So $\mathcal{Z}_{t}^{1}\left(\mathcal{N}\left(\ell_{p}(G)\right)^{* *}\right) \subseteq \mathcal{N}\left(\ell_{p}(G)\right)$ and hence

$$
\mathcal{Z}_{t}^{1}\left(\mathcal{N}\left(\ell_{p}(G)\right)^{* *}\right)=\mathcal{N}\left(\ell_{p}(G)\right)
$$

This extends Neufang's result (Theorem 6.1.1) when $G$ is discrete, here we do not need to assume that $G$ is second countable.

What Lemma 6.4.1 says is that $\left\{\psi_{ \pm}\right\} \subseteq \operatorname{LUC}(G)^{*}$ is, in some sense, determining for the first topological centre of $\mathcal{N}\left(L_{p}(G)\right)$. So we propose the following definition:

Definition 6.4.3. Let $\mathfrak{A}$ be a Banach algebra, $\lambda$ be a representation of $\left(\mathfrak{A}^{* *}, \square\right)$ on some Banach space $X$ and $\rho$ be representation of some other algebra $\mathfrak{A}^{\prime}$ on $X$ s.t.

$$
m \in \mathcal{Z}_{t}^{1}\left(\mathfrak{A}^{* *}\right) \Longleftrightarrow \lambda(m) \rho(a)=\rho(a) \lambda(m) \quad \forall a \in \mathfrak{A}^{\prime} .
$$

Then $\mathfrak{D} \subseteq \mathfrak{A}^{\prime}$ is $(\lambda, \rho)-L D T C$ for $\mathfrak{A}$ if

$$
\lambda(m) \rho(d)=\rho(d) \lambda(m) \quad \forall d \in \mathfrak{D} \quad \Rightarrow \quad m \in \mathcal{Z}_{t}^{1}\left(\mathfrak{A}^{* *}\right) .
$$

$(\lambda, \rho)$-RDTC sets are defined by replacing $\square$ with $\diamond$ and $\mathcal{Z}_{t}^{1}$ with $\mathcal{Z}_{t}^{2}$

This definition is not entirely satisfactory since the representations $\lambda$ and $\rho$ are not canonical, as in the case of $\mathcal{N}\left(\ell_{p}(G)\right)$, but it allows us to state Lemma 6.4.1 in the following way.

Theorem 6.4.4. $\left\{\psi_{ \pm}\right\} \subseteq \operatorname{LUC}(G)^{*}$ is a $(\Gamma, \tilde{\gamma})-L D T C$ set for $\mathcal{N}\left(\ell_{p}(G)\right)$. 
Of course, if $G$ is abelian, we have a 2-point DTC set in the sense of Definition 2.3.5.

Theorem 6.4.5. If $G$ is infinite abelian discrete, then $\mathcal{N}\left(\ell_{p}(G)\right)^{* *}$ is 2-LDTC.

Proof. Let $m \in \mathcal{N}\left(\ell_{p}(G)\right)^{* *}$ and suppose that

$$
m \square \widehat{\psi_{ \pm}}=m \diamond \widehat{\psi_{ \pm}}
$$

Write

$$
\psi_{ \pm}=w^{*}-\lim _{\alpha} \mu_{ \pm \alpha}
$$

with the $\mu_{ \pm \alpha} \in M(G)$. Then

$$
w^{*}-\lim _{\alpha} \widehat{\mu_{ \pm \alpha}}=\widehat{\psi_{ \pm}}
$$

so by assumption

$$
m \square w^{*}-\lim _{\alpha} \widehat{\mu_{ \pm \alpha}}=w^{*}-\lim _{\alpha} m \square \widehat{\mu_{ \pm \alpha}}
$$

Then since $G$ is abelian, by Theorem 6.3 .3 ,

$$
\Gamma(m) \tilde{\gamma}\left(\psi_{ \pm}\right)=\tilde{\gamma}\left(\psi_{ \pm}\right) \Gamma(m)
$$

Then by Lemma 6.4.1 and Theorem 6.4.2,

$$
m \in \mathcal{N}\left(\ell_{p}(G)\right)=\mathcal{Z}_{t}^{1}\left(\mathcal{N}\left(\ell_{p}(G)\right)^{* *}\right)
$$

Thus $\left\{\widehat{\psi_{ \pm}}\right\}$is a left DTC set for $\mathcal{N}\left(\ell_{p}(G)\right)$.

This DTC result is actually stronger than the definition of DTC sets (Definition 2.3.5) requires. We defined DTC sets in terms of all nets converging $w^{*}$ to elements 
of the DTC set, whereas we only needed $w^{*}$ continuity against one net converging to each of the determining elements.

In general, we only have a left DTC set consisting of 2 times $\left|\ell_{1}(G)\right|$ many points. Although this DTC set is infinite, it consists of the two points $\widehat{\psi_{ \pm}}$blown up by "good" elements $\widehat{\pi(\rho)} \in \mathcal{Z}_{t}^{1}\left(\mathcal{N}\left(\ell_{p}(G)\right)^{* *}\right)$ (by Remark 6.2.5).

Theorem 6.4.6. Let $G$ be infinite discrete. Then

$$
\mathfrak{D}:=\left\{\widehat{\pi(\rho)} \square \widehat{\psi_{ \pm}} \mid \rho \in \mathcal{N}\left(\ell_{p}(G)\right)\right\}=\left\{\widehat{h} \square \widehat{\psi_{ \pm}} \mid h \in \ell_{1}(G)\right\}
$$

is a left DTC set for $\mathcal{N}\left(\ell_{p}(G)\right)$.

Proof. Let $m \in \mathcal{N}\left(\ell_{p}(G)\right)^{* *}$ and suppose that for every net $n_{\alpha}$ in $\mathcal{N}\left(\ell_{p}(G)\right)^{* *}$ converging $w^{*}$ to some $d=\widehat{\pi(\rho)} \square \widehat{\psi_{ \pm}} \in \mathfrak{D}$

$$
m \square w^{*}-\lim _{\alpha} n_{\alpha}=w^{*}-\lim _{\alpha} m \square n_{\alpha} .
$$

If $\widehat{\psi_{ \pm}}=w^{*}-\lim _{\alpha} \widehat{\mu_{\alpha}}$ with the $\mu_{\alpha} \in M(G)$ then

$$
w^{*}-\lim _{\alpha} \widehat{\pi(\rho)} \square \widehat{\mu_{\alpha}}=\widehat{\pi(\rho)} \square w^{*}-\lim _{\alpha} \widehat{\mu_{\alpha}}=\widehat{\pi(\rho)} \square \widehat{\psi_{ \pm}}=d
$$

and hence in particular

$$
m \square w^{*}-\lim _{\alpha} \widehat{\pi(\rho)} \square \widehat{\mu_{\alpha}}=w^{*}-\lim _{\alpha} m \square \widehat{\pi(\rho)} \square \widehat{\mu_{\alpha}} .
$$

So by Lemma 6.3.1,

$$
\Gamma(m) \tilde{\gamma}\left(\psi_{ \pm}\right)=\tilde{\gamma}\left(\psi_{ \pm}\right) \Gamma(m)
$$


Then by Lemma 6.4.1 and Theorem 6.4.2,

$$
m \in \mathcal{N}\left(\ell_{p}(G)\right)=\mathcal{Z}_{t}^{1}\left(\mathcal{N}\left(\ell_{p}(G)\right)^{* *}\right)
$$

\subsection{A right DTC set}

Compared to how hard we had to work for left DTC sets for $\mathcal{N}\left(\ell_{p}(G)\right)$, we get right ones almost for free from [3] and [11], and we do not need to worry about alternate notions of being determining for the topological centre.

The identifications in Section 6.1 allow us to lift right DTC sets for $\ell_{1}(G)$ to right DTC sets for $\mathcal{N}\left(\ell_{p}(G)\right)$.

Theorem 6.5.1. Let $\mathfrak{D}$ be a right $D T C$ set for $\ell_{1}(G)$. Then $\mathfrak{D}$ is a right DTC set for $\mathcal{N}\left(\ell_{p}(G)\right)$, identifying $\mathfrak{D}$ with

$$
\{(d, 0): d \in \mathfrak{D}\} \subseteq \ell_{1}(G)^{* *} \oplus M_{\ell_{\infty}(G)}^{\perp}=\mathcal{N}\left(\ell_{p}(G)\right)^{* *}
$$

Proof. Write $m=(h, \Xi) \in \ell_{1}(G)^{* *} \oplus M_{\ell_{\infty}(G)}^{\perp}=\mathcal{N}\left(\ell_{p}(G)\right)^{* *}$ and suppose that

$$
d \square m=d \diamond m \quad \forall d \in \mathfrak{D} .
$$

Then

$$
\begin{aligned}
(d, 0) \boldsymbol{\beta}(h, \Xi) & =(d, 0) \boldsymbol{\phi}(h, \Xi) \\
(d \square h, d \square \Xi) & =(d \diamond h, d \diamond \Xi) \\
\Rightarrow d \square h & =d \diamond h .
\end{aligned}
$$


Then since $\mathfrak{D}$ is a right DTC set for $\ell_{1}(G)$,

$$
h \in \mathcal{Z}_{t}^{2}\left(\ell_{1}(G)^{* *}\right)=\ell_{1}(G) \quad \Rightarrow \quad(h, \Xi) \in \ell_{1}(G) \oplus M_{\ell_{\infty}(G)}^{\perp}=\mathcal{Z}_{t}^{2}\left(\mathcal{N}\left(L_{p}(G)\right)^{* *}\right)
$$

So $\mathfrak{D}$ is a right DTC set for $\mathcal{N}\left(L_{p}(G)\right)$.

Recalling that $\ell_{1}(G)$ has a 2-point right DTC set (Theorem 12.22 in [11]),

Corollary 6.5.2. $\mathcal{N}\left(\ell_{p}(G)\right)$ is $2-R D T C$. 


\section{References}

[1] R. Arens. The adjoint of a bilinear operation. Proc. Am. Math. Soc., 2:839-848, 1951.

[2] R. Arens. Operations induced in function classes. Monatsh. Math., 55:1-19, 1951.

[3] C. Auger. Jordan-Arens irregularity. Master's thesis, Carleton University, 2007.

[4] M. E. B. Bekka. Mittelbare unitäre Darstellung lokalkompakter Gruppen. Habilitation Techn. Univ. München, 1989.

[5] M. E. B. Bekka. Amenable unitary representations of locally compact groups. Invent. Math., 100:383-401, 1990.

[6] P. Civin and B. Yood. The second conjugate space of a Banach algebra as an algebra. Pacific J. Math, 11:847-870, 1961.

[7] D. L. Cohn. Measure Theory. Birkhäuser Boston, Boston, 1993. Reprint of the 1980 original.

[8] H. G. Dales. Banach Algebras and Automatic Continuity. Oxford University Press, Oxford, 2000.

[9] H. G. Dales, F. Gharahmani, and N. Grønbæk. Derivations into iterated duals of Banach algebras. Studia Math., 128:19-54, 1998.

[10] H. G. Dales and A. T.-M. Lau. The second duals of Beurling algebras. Memoirs of the American Mathematical Society, 177, 2005.

[11] H. G. Dales, A. T.-M. Lau, and D. Strauss. Banach Algebras on Semigroups and their Compactifications. Submitted to Memoirs of the American Mathematical Society.

[12] P. Enflo. A counterexample to the approximation problem in Banach spaces. Acta Math., 130:309-317, 1973. 
REFERENCES

[13] M. Fabian et al. Functional Analysis and Infinite-Dimensional Geometry. Springer-Verlag, New York, 2001.

[14] G. B. Folland. A Course in Abstract Harmonic Analysis. CRC Press, Boca Raton, FL, 1995.

[15] F. Ghahramani. Isometric representation of $M(G)$ on $B(H)$. Glasg. Math. J., 23:119-122, 1982.

[16] M. Grosser and V. Losert. The norm-strict bidual of a Banach algebra and the dual of $C_{u}(G)$. Manuscripta Math., 45(2):127-146, 1984.

[17] A. Grothendieck. Produits tensoriels topologiques et espaces nucléaires. Memoirs of the American Mathematical Society, 16, 1955.

[18] P. R. Halmos. Finite-Dimensional Vector Spaces. Springer-Verlag, New York, 1974. Reprint of the 1942 original.

[19] E. Hewitt and K. A. Ross. Abstract Harmonic Analysis II. Springer-Verlag, New York, 1970.

[20] E. Hewitt and K. A. Ross. Abstract Harmonic Analysis I. Springer-Verlag, New York, second edition, 1979.

[21] H. Hofmeier and G. Wittstock. A bicommutant theorem for completely bounded module homomorphisms. Math. Ann., 308:141-154, 1997.

[22] T. Kappeler. Banach spaces with the Condition of Mazur. Math. Z., 191:623631, 1986.

[23] J. L. Kelley. General Topology. Springer-Verlag, New York, 1975. Reprint of the 1955 original.

[24] A. T.-M. Lau. Continuity of Arens multiplication on the dual space of bounded uniformly continuous functions on locally compact groups and topological semigroups. Math. Proc. Camb. Phil. Soc., 99:273-283, 1986.

[25] A. T.-M. Lau and V. Losert. On the second conjugate algebra $L_{1}(G)$ of a locally compact group. J. London Math. Soc., 37:464-470, 1988.

[26] R. E. Megginson. An Introduction to Banach Space Theory. Springer-Verlag, New York, 1998.

[27] M. Neufang. The algebra $X(G)^{*}$. Preprint.

[28] M. Neufang. One-sided strong Arens irregularity. Preprint.

[29] M. Neufang. A two-point dtc set for $\operatorname{LUC}(G)^{*}$. Preprint. 
[30] M. Neufang. Abstrakte Harmonische Analyse und Modulhomomorphismen über von Neumann-Algebren. PhD thesis, Universität des Saarlandes, 2000.

[31] T. W. Palmer. Banach Algebras and the General Theory of*-Algebras, Volume I: Algebras and Banach Algebras. Cambridge University Press, Cambridge, 1994.

[32] V. Runde. Amenability for dual Banach algebras. Studia. Math., 148:47-66, 2001.

[33] R. A. Ryan. Introduction to Tensor Products of Banach Spaces. Springer-Verlag, New York, 2002.

[34] H. H. Schaefer and M. Wolff. Topological Vector Spaces. Springer-Verlag, New York, second edition, 1999.

[35] S. Willard. General Topology. Dover Publications, New York, 2004. Reprint of the 1970 original. 